M. Weber

Nagoya Math. J.

Vol. 118 (1990), 65-97

\title{
THE LAW OF THE ITERATED LOGARITHM ON SUBSEQUENCES-CHARACTERIZATIONS
}

\author{
MICHEL WEBER
}

Let $;$ be any increasing sequence of integers and $M>1$; we connect to them in a very simply way, an increasing unbounded function $\varphi:$ $\boldsymbol{R}^{+}$. Let also $X_{1}, X_{2}, \cdots$ be a sequence of i.i.d. random vectors with value in euclidian space $R^{m}$. We prove that the cluster set of the sequence $\left\{\left(X_{1}+\cdots+X_{n}\right) / \sqrt{n} \varphi(n), n \in ?\right\}$ almost surely coincides with the unit ball of $\boldsymbol{R}^{m}$, if, and only if, the covariance matrix of $X_{1}$ is the identity matrix of $\boldsymbol{R}^{m}$ and $E X_{1}$ is the zero vector of $\boldsymbol{R}^{m}$. We define a functional $A$ on the set of increasing sequences of integers as follows:

$$
A(:)=\limsup _{j \rightarrow \infty}\left\{\frac{\log \#\left(i \leq j: \vdots \cap\left[M^{i}, M^{i+1}[\neq \varnothing)\right.\right.}{\log j}\right\}^{1 / 2} .
$$

We prove that $P\left\{\limsup _{\text {เə } n \rightarrow \infty}\left(X_{1}+\cdots+X_{n}\right) / \sqrt{ } 2 n \log \log n>0\right\}>0$, for at least one sequence $X_{1}, X_{2}, \cdots$ of i.i.d. real r.v.'s with $E X_{1}=0$ and $E\left(X_{1}\right)^{2}$ $<\infty$, if, and only if $A(\vec{S})>0$; further the definition of $A(\cdot)$ does not depend of the value of $M$. Different characterizations are also established. Further, the law of the iterated logarithm in the sense of Strassen is considered. We finally show a functional law of the iterated logarithm on subsequences for lipschitzian random functions.

\section{$\S 1$. Introduction}

This work is a natural continuation of our previous results obtained in [16]. Let $X_{1}, X_{2}, \cdots$ be a sequence of independent, identically distributed (i.i.d), real random variables (r.v.'s). Set

$$
\forall n \geq 1, \quad S_{n}=X_{1}+\cdots+X_{n} .
$$

We investigate the asymptotic behavior of the sequence $\left\{S_{n}\right\}$ when $n$ runs on arbitrary subsequences of integers. This is a quite natural

Received November 28, 1988. 
question since it is well-known that, when $E X_{1}=0, E\left(X_{1}\right)^{2}=1$,

$$
P\left\{\limsup _{k \rightarrow \infty} \frac{S_{2^{k}}}{\sqrt{2.2^{k} \log \log 2^{k}}}=1\right\}=1 \text {. }
$$

In fact, it turns out that for any $\delta \geq 1$, letting

$$
\mathfrak{g}_{\delta}=\left\{2^{[n \delta]}, n \geq 1\right\},
$$

where we write $[x]$ the integer part of $x$,

$$
P\left\{\limsup _{\delta_{\delta} \ni n \rightarrow \infty} \frac{S_{n}}{\sqrt{2 \mathrm{n} \log \log n}}=\delta^{-1 / 2}\right\}=1,
$$

so that (1.1) appears as a particular case $(\delta=1)$ of a much more general phenomenon. From this, we can also deduce the classical HartmannWintner law of the iterated logarithm [7] under a stronger form.

Let now $\xi$ be any strictly increasing sequence of integers and $M>1$. In a previous work, we connected to $\mathfrak{s}$ and $M$, in a very simple way a function $\varphi: \mathfrak{Z} \rightarrow \boldsymbol{R}^{+}$such that for every sequence $X_{1}, X_{2}, \cdots$ of i.i.d. real r.v.'s satisfying $E X_{1}=0, E\left(X_{1}\right)^{2}=1$,

$$
P\left\{C_{1} \leq \limsup _{\beta \ni n \rightarrow \infty} \frac{S_{n}}{\sqrt{n} \varphi(n)} \leq C_{2}\right\}=1,
$$

where $0<C_{1} \leq C_{2}<\infty$ depends on $M$ only. One of our first results in this work (see Theorem 2.1) is that $C_{1}=C_{2}=1$ without any modification nor restriction. Conversely, if $X_{1}, X_{2}, \cdots$ is a sequence of i.i.d. real r.v.'s such that (1.3) holds $\left(C_{1}=C_{2}=1\right)$, for some triple $(\xi, M, \varphi)$ defined above, then

$$
E X_{1}=0 \text { and } E\left(X_{1}\right)^{2}=1 .
$$

Further, this characterization extends to the case of any sequence of i.i.d. random vectors with values in euclidian spaces. This is the Theorem 2.1. The cluster set of the sequence $\left\{\frac{S_{n}}{\sqrt{n} \varphi(n)}, n \in \mathfrak{g}\right\}$ is also identified, so that the law of the iterated logarithm on any subsequence, in euclidian spaces is characterized. Besides, we characterize all the subsequences $\mathfrak{g}$ such that

$$
P\left\{\limsup _{3 \ni n \rightarrow \infty} \frac{X_{1}+\cdots+X_{n}}{\sqrt{2 n \log \log n}}>0\right\}>0,
$$

for at least one sequence $X_{1}, X_{2}, \cdots$ of i.i.d. real r.v.'s such that $E X_{1}=0$ 
and $E\left(X_{1}\right)^{2}<\infty$. This is the Theorem 2.3. Moreover, we generalize this result in Theorem 2.8. These results are proved in Section 3. Fix now any strictly increasing sequence of integers $\mathcal{F}=\left\{n_{k}, k \geq 1\right\}$ and $M>1$. Let $\eta_{N} \in C([0,1])$ be obtained by linearly interpolating the partials sums $S_{n_{k}}$ at $n_{k} / n_{N}, 1 \leq k \leq N, N \geq 1$. In Theorem 2.9, we determine the cluster set of the sequence

$$
\left\{\frac{\eta_{N}}{\sqrt{n_{N}} \varphi\left(n_{N}\right)}, N \geq 1\right\}
$$

in two particular cases, and therefore we show that this cluster set changes very much with the sequence 3 . This is proved in Section 4 . The main feature of the law of the iterated logarithm on subsequences in euclidian spaces, is contained in the fact that the behavior of the partial sums, when indexed on subsequences, can be as small as we want, (see $\left(2.2^{\prime}\right)$ ). In Theorem 2.10, we show that this feature is preserved in infinite dimension. This result is proved in Section 5 .

\section{$\S 2$. Notations, main results}

Let $\mathfrak{B}=\left\{n_{k}, k \geq 1\right\}$ be any strictly increasing sequence of integers and $M>1$. We associate to them, (as in [16]),

$$
\left\{\begin{array}{l}
I_{0}=I_{0}(M)=\left[0, M\left[\text { and for each integer } k \geq 1, I_{k}=I_{k}(M)=\right.\right. \\
{\left[M^{k}, M^{k+1}\right], \text { for every } p \geq 0, \delta_{p}=\delta_{p}(\mathfrak{g}, M)=\left\{\begin{array}{l}
1 \text { if } \mathfrak{g} \cap I_{p}(M) \neq \varnothing, \\
0 \text { unless, }
\end{array}\right.} \\
k_{1}=k_{1}(\mathfrak{g}, M)=\inf \left\{n \geq 0: \delta_{n}(\mathfrak{s}, M)=1\right\}, \text { and for every } p>1, \\
k_{p}=k_{p}(\mathfrak{s}, M)=\inf \left\{n \geq k_{p-1}: \delta_{n}(\mathfrak{s}, M)=1\right\}, \\
\text { for each } p \geq 1, n_{p}^{*} \text { is some point of } \mathfrak{g} \cap I_{k_{p}}(M) \text { and } \mathfrak{g}^{*}=\left\{n_{q}^{*}, p \geq 1\right\}, \\
\text { for every } n \in \mathfrak{s}, \varphi(n)=\varphi(\mathfrak{s}, M, n)=\sqrt{2 \log (p+2)} \text { iff } n \in I_{k_{p}}(M) .
\end{array}\right.
$$

Afterwards, a triple $(\mathfrak{s}, M, \varphi)$ will be always composed of a strictly increasing sequence of integers, a number $M$ strictly greater than 1 and a $\operatorname{map} \varphi: \mathfrak{s} \rightarrow \boldsymbol{R}^{+}$, defined in accordance with the notations (2.1). Note that $\varphi$ is depending on $\xi$ and $M$. Let $\psi: N \rightarrow N$ be strictly increasing and set

$$
\mathfrak{s}(\psi)=\left\{2^{\psi(k)}, k \geq 1\right\}
$$

Then, for every $M \geq 1$,

$$
\lim _{\mathscr{B}(\psi) \ni n \rightarrow \infty} \frac{\varphi(\mathfrak{g}(\psi), M, n)}{\sqrt{2 \log \psi^{-1}(\log \log n)}}=1
$$


so that, when $\psi$ grows very fast, the corresponding function $\varphi$ grows very slowly. Our first result characterizes the law of the iterated logarithm on subsequences in euclidian spaces.

THEOREM 2.1. Let $(\mathfrak{s}, M, \varphi)$ be any triple defined in accordance with (2.1), and a sequence $X=\left\{X_{i}, i \geq 1\right\}$ of i.i.d. random vectors with value in $m$-dimensional euclidian space $\boldsymbol{R}^{m}$. Let $B_{m}$ be the unit ball of $\boldsymbol{R}^{m}$ and set

$$
\forall n \geq 1, \quad S_{n}(X)=X_{1}+\cdots+X_{n} .
$$

Then,

$$
P\left\{C\left(\left\{\frac{S_{n}(X)}{\sqrt{n} \varphi(n)}, n \in \mathfrak{Z}\right\}\right)=B_{m}\right\}=1
$$

if, and only if.

(2.4) $\operatorname{Cov}\left(X_{1}\right)$ is the identity matrix of $\boldsymbol{R}^{m}$ and $E X_{1}$ the zero vector of $\boldsymbol{R}^{m}$. In particular, when $m=1$.

$$
P\left\{\limsup _{\nexists \ni n \rightarrow \infty} \frac{S_{n}(X)}{\sqrt{n} \varphi(n)}=1\right\}=1,
$$

if, and only if,

$$
E X_{1}=0 \text { and } E\left(X_{1}\right)^{2}=1
$$

Further

$$
P\left\{C\left(\left\{\frac{S_{n}(X)}{\sqrt{n} \varphi(n)}, n \in \mathfrak{g}\right\}\right)=[-1,1]\right\}=1
$$

This characterization is proved in Section 3. Once it is proved for $m=1$, the general case is easily deduced from the work of $H$. Finkelstein [5], for the sufficienty part. The necessity part is obtained by choosing suitable linear forms $f_{i, j} \in\left(\boldsymbol{R}^{m}\right), i, j=1, \cdots, m$, and applying the result obtained for $m=1$ to the sequences $\left\{f_{i, j}\left(X_{n}\right), n \in \mathfrak{B}\right\}$. The proof of Theorem 2.1, when $m=1$, depends essentially of the following intermediate result:

Proposition 2.2. Let $(\mathfrak{B}, M, \varphi)$ be any triple defined in accordance with (2.1) and $\delta \geq 1$. Set,

$$
\mathfrak{G}_{\delta}^{*}=\left\{n_{\left[p^{\delta}\right]}^{*}, p \geq 1\right\}
$$


where $[x]$ is the integer part of $x$. Then, for any sequence $X_{1}, X_{2}, \cdots$ of i.i.d. real r.v.'s satisfying $E X_{1}=0$ and $E\left(X_{1}\right)^{2}=1$,

$$
P\left\{\limsup _{\mathbb{B}_{\delta}^{*} \ni n \rightarrow \infty} \frac{S_{n}(X)}{\sqrt{n} \varphi(n)}=(\delta)^{-1 / 2}\right\}=1 .
$$

This is precisely the intermediate step (3.59) in the proof of lemma 3.4; further (2.9) easily implies (2.7). One sees in particular, when $\delta=1$, that the lower bound in (2.5) is reached on $\mathfrak{s}^{*}$.

Next, we define on the set of strictly increasing sequences of integers, a functional as follows: let $M>1$, then we put:

$$
\Lambda(\mathfrak{g})=\limsup _{j \rightarrow \infty}\left\{\frac{\log \sharp\left(i \leq j: \mathfrak{g} \cap\left[M^{i}, M^{i+1}[\neq \varnothing)\right.\right.}{\log j}\right\}^{1 / 2} .
$$

It is easily seen that

$$
\Lambda(\mathfrak{g})=\limsup _{j \rightarrow \infty}\left\{\frac{\log j}{\log k_{j}(\mathfrak{g}, M)}\right\}^{1 / 2},
$$

where we use the notations (2.1).

Note that $0 \leq \Lambda(\mathfrak{g}) \leq 1$ and $\Lambda(\mathfrak{g})$, a priori, depends on the value of $M>1$. This functional will give us the possibility to characterize in a very simple way the sequences of integers which "support" the law of the iterated logarithm.

TheOREM 2.3. Let $X_{1}, X_{2}, \cdots$ be any sequence of i.i.d. real r.v.'s satisfying $E X_{1}=0$ and $E\left(X_{1}\right)^{2}=1$. Then, for any strictly increasing sequence of integers $\mathcal{B}$,

$$
P\left\{\limsup _{i \ni n \rightarrow \infty} \frac{X_{1}+\cdots+X_{n}}{\sqrt{2 n \log \log n}}>0\right\}>0
$$

if, and only if,

$$
\Lambda(\mathfrak{g})>0
$$

Then, we have,

$$
P\left\{\limsup _{\Re \ni n \rightarrow \infty} \frac{X_{1}+\cdots+X_{n}}{\sqrt{2 n \log \log n}}=\Lambda(\mathfrak{g})\right\}=1 .
$$

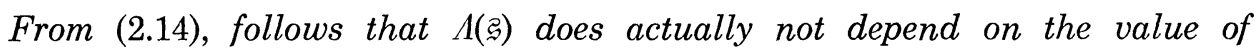
$M>1$. This result takes a very simple form when the sequence $\mathfrak{B}$ is of the type defined in (2.2). 
COROLlary 2.4. Let $X_{1}, X_{2}, \cdots$ be a sequence of i.i.d. r.v.'s satisfying $E X_{1}=0$ and $E\left(X_{1}\right)^{2}=1$. Then, for any strictly increasing map $\psi: N \rightarrow N$,

$$
P\left\{\limsup _{z(\psi) \ni n \rightarrow \infty} \frac{X_{1}+\cdots+X_{n}}{\sqrt{2 n \log \log n}}=\limsup _{m \rightarrow \infty}\left[\frac{\log m}{\log \psi(m)}\right]^{1 / 2}\right\}=1,
$$

where the sequence $\mathfrak{g}(\psi)$ is defined in (2.2).

In our next approach, we collect some easy properties of the functional $\Lambda(\cdot)$. We set for any integer $r \geq 1, \alpha>0$ and any increasing sequence of integers $\mathfrak{Z}$,

$$
\left\{\begin{array}{l}
P_{\alpha}(\mathfrak{I})=\left\{\left[n^{\alpha}\right], n \in \mathfrak{S}\right\} \quad \text { and } P_{\alpha}=P_{\alpha}(N), \\
2^{\mathfrak{Z}}=\left\{2^{n}, n \in \mathfrak{S}\right\}, \\
\mathfrak{S}^{(r)}=\left\{n_{1}+\cdots+n_{r}, \forall i=1, \cdots r, n_{i} \in \mathfrak{S}\right\}, \\
\alpha \mathfrak{S}=\{[\alpha n], n \in \mathfrak{S}\} .
\end{array}\right.
$$

LEMma 2.5. The following properties hold for any $\alpha>0$ and any increasing sequence of integers $\xi$,

a) $\Lambda(\alpha \mathfrak{s})=\Lambda\left(P_{\alpha}(\mathfrak{s})\right)=\Lambda(\mathfrak{s})$,

b) $\Lambda\left(2^{8}\right)=\Lambda\left(2^{\alpha \beta}\right)$,

c) if $\mathfrak{g}_{i}=\left\{n_{k}^{i}, k \geq 1\right\}, i=1,2$ satisfy

$$
\begin{aligned}
& 0<\liminf _{k \rightarrow \infty} n_{k}^{1} / n_{k}^{2} \leq \limsup _{k \rightarrow \infty} n_{k}^{1} / n_{k}^{2}<\infty, \\
& \text { then, } \Lambda\left(\mathfrak{F}_{1}\right)=\Lambda\left(\mathfrak{F}_{2}\right) .
\end{aligned}
$$

We notice that $\mathrm{b}$ ) expresses the fact that $\Lambda(\cdot)$ does actually not depend of the number $M>1$ used in its definition; further a) follows easily from b). As for c), since there are constants $0<C_{1} \leq C_{2}<\infty$, such that for all $k$,

$$
C_{1} \leq n_{k}^{1} / n_{k}^{2} \leq C_{2}
$$

we therefore have $\#\left\{i \leq n: \mathfrak{g}_{1} \cap\left[M^{i}, M^{i+1}[\neq \varnothing\} \leq \inf \left\{p: M^{n} \leq C_{1} M^{k_{p}\left(z_{2}, M\right)}\right\}\right.\right.$, for all $n$, and this easily leads to $\Lambda\left(\mathfrak{F}_{1}\right) \leq \Lambda\left(\mathfrak{F}_{2}\right)$, which implies c) by symmetry.

Let now $\xi=\left\{\xi_{n}, n \geq 1\right\}$ be an increasing sequence of integer valued r.v.'s such that

$$
P\left\{\lim _{k \rightarrow \infty} \frac{\xi_{k}}{n_{k}}=1\right\}=1
$$

for some increasing sequence $\mathfrak{g}=\left\{n_{k}, k \geq 1\right\}$. 
From the previous lemma, one deduces

$$
P\left\{\Lambda\left(\left\{\xi_{n}, n \geq 1\right\}\right)=\Lambda(\xi)\right\}=1 .
$$

Therefore, putting together Theorem 2.3 and (2.18) leads to

Corollary 2.6. Let $\mathfrak{g}=\left\{n_{k}, k \geq 1\right\}$ be an increasing sequence of integers and an increasing sequence $\xi=\left\{\xi_{k}, k \geq 1\right\}$ of integer valued r.v.'s related to $\xi$ by (2.17). Then, for any sequence $X_{1}, X_{2}, \cdots$ of i.i.d. real r.v.'s with $E X_{1}=0, E\left(X_{1}\right)^{2}=1$, which is independent of the sequence $\xi$, one has

$$
P\left\{\limsup _{k \rightarrow \infty} \frac{\sum_{i=1}^{\xi_{k}} X_{i}}{\sqrt{2} n_{k} \log \log n_{k}}=\Lambda(\mathfrak{s})\right\}=1 \text {. }
$$

The next corollary establishes some relation between the law of the iterated logarithm and the classical Waring's problem in additive number theory, (see e.g. [15], Chapter I).

Corollary 2.7. Let $r, q$ be two positive integers. For any sequence $X_{1}, X_{2}, \cdots$ of i.i.d. real r.v.'s satisfying $E X_{1}=0$ and $E\left(X_{1}\right)^{2}=1$,

$$
\begin{aligned}
& P\left\{\limsup _{2^{(r)} \ni n \rightarrow \infty} \frac{X_{1}+\cdots+X_{n}}{\sqrt{2 n \log \log n}}\right. \\
& \left.\quad=\limsup _{N \rightarrow \infty}\left[\frac{\log \#\left\{n=i_{1}^{q}+\cdots+i_{r}^{q} \leq N\right\}}{\log N}\right]^{1 / 2}\right\}=1,
\end{aligned}
$$

where we use the notation (2.16).

This raises the following question: to determine the asymptotic behavior of

$$
\left\{\left(X_{1}+\cdots+X_{n}\right) / \sqrt{2 n \log \log n}, n \in 2^{8(r)}\right\}
$$

with respect to those of

$$
\left\{\left(X_{1}+\cdots+X_{n}\right) / \sqrt{2 n \log \log n}, n \in 2^{8}\right\} .
$$

Indeed, the fundamental aspect of the Waring's problem being solved (see again [15]), we know that

$$
\forall q \text { integer, } \exists r=r(q)<\infty \text { such that } P_{q}^{(r)}=N,
$$

in other words each sequence $P_{q}$ defines a natural basis of the integers. For instance, if $q=2$

$$
P_{2}^{(4)}=N
$$


so that,

$$
P\left\{\limsup _{P_{2}^{(1)} \ni n \rightarrow \infty} \frac{X_{1}+\cdots+X_{n}}{\sqrt{2 n \log \log n}}=(2)^{-1 / 2}\right\}=1,
$$

whereas,

$$
P\left\{\limsup _{P_{2}^{(4)} \ni n \rightarrow \infty} \frac{X_{1}+\cdots+X_{n}}{\sqrt{2 n \log \log n}}=1\right\}=1 .
$$

We thus can wonder what becomes (2-20) when indexing the partials sums on $P_{2}^{(2)}$ or $P_{2}^{(3)}$. We have not been able to answer this question. The problem solved by Theorem 2.3 extends immediately: let $(\mathfrak{z}, M, \varphi)$ be a triple defined in accordance with (2.1); characterize all the subsequences, $\mathfrak{F}_{1} \subset \mathfrak{g}$, such that

$$
P\left\{0<\limsup _{\delta_{1} \ni n \rightarrow \infty} \frac{X_{1}+\cdots+X_{n}}{\sqrt{n} \varphi(n)} \leq 1\right\}>0,
$$

for at least one sequence $X_{1}, X_{2}, \cdots$ of i.i.d. real $r, v$.'s satisfying $E X_{1}=0$, $E\left(X_{1}\right)^{2}=1$. Put,

$$
A\left(\mathfrak{g}, M, \mathfrak{g}_{1}\right)=\limsup _{j \rightarrow \infty}\left[\frac{\log \sharp\left\{i \leq j: \mathfrak{s}_{1} \cap\left[M^{i}, M^{i+1}[\neq \varnothing\}\right.\right.}{\log \sharp\left\{i \leq j: \mathfrak{s} \cap\left[M^{i}, M^{i+1}[\neq \varnothing\}\right.\right.}\right]^{1 / 2} .
$$

We have the following characterization

THEOREM 2.8. Let $(\mathcal{S}, M, \varphi)$ be any triple defined in accordance with the notation (2.1). Then, for any sequence $X_{1}, X_{2}, \cdots$ of i.i.d. real r.v.'s $E X_{1}=0, E\left(X_{1}\right)^{2}=1$, and any subsequence $\mathfrak{g}_{1} \subset \mathfrak{g}$,

$$
P\left\{\limsup _{\mathfrak{s}_{1} \ni n \rightarrow \infty} \frac{X_{1}+\cdots+X_{n}}{\sqrt{n} \varphi(n)}=\Lambda\left(\mathfrak{s}, M, \mathfrak{I}_{1}\right)\right\}=1 .
$$

This theorem is proved in Section 3. Its proof as well as proof of Theorem 2.3, depends essentially of the Theorem 3.3.

Fix now a triple $(\mathfrak{B}, M, \varphi)$ according to the notation (2.1) with $\mathfrak{g}=$ $\left\{n_{k}, k \geq 1\right\}$, as well as a sequence $X_{1}, X_{2}, \cdots$ of i.i.d. real r.v.'s satisfying $E X_{1}=0, E\left(X_{1}\right)^{2}=1$. Let $\eta_{N} \in \underline{C}([0,1]), N \geq 1$, be obtained by linearly interpolating the partial sums

$$
S_{n_{k}}=X_{1}+\cdots+X_{n_{k}}
$$

at points $n_{k} / n_{N}, 1 \leq k \leq N$. Thus, (with the convention $S_{0}=0$ ) 


$$
\left\{\begin{array}{l}
\forall t \in[0,1], \eta_{N}(t)=S_{n_{k}}\left[1+\frac{n_{k}-t n_{N}}{n_{k+1}-n_{k}}\right]+S_{n_{k+1}}\left[\frac{t n_{N}-n_{k}}{n_{k+1}-n_{k}}\right] \\
\text { if } n_{k} \leq t n_{N} \leq n_{k+1} .
\end{array}\right.
$$

We put,

$$
\left\{\begin{array}{l}
K_{1}=\left\{f(t)=\int_{0}^{t} g(u) d u: \int_{0}^{1} g^{2}(u) d u \leq 1\right\}, \\
K_{2}=\{f(t)=\varepsilon t \quad \varepsilon \in[-1,1]\}
\end{array}\right.
$$

The next result states a law of the iterated logarithm in the sense of $V$. Strassen [12], for subsequences.

THEOREM 2.9. Let $(\xi, M, \varphi)$ be any triple defined in accordance with (2.1), and a sequence $X_{1}, X_{2}, \cdots$ of i.i.d. real r.v.'s satisfying $E X_{1}=0$ and $E\left(X_{1}\right)^{2}=1$.

a) if $\lim _{k \rightarrow \infty} n_{k} / n_{k+1}=1$, then

$$
P\left\{\lim _{N \rightarrow \infty} \operatorname{dist}\left(\eta_{N} / \sqrt{n_{N}} \varphi\left(n_{N}\right), K_{1}\right)=0\right\}=1,
$$

and

$$
P\left\{C\left(\left\{\frac{\eta_{N}}{\sqrt{n_{N}} \varphi\left(n_{N}\right)}, N \geq 1\right\}\right)=K_{1}\right\}=1
$$

b) if $\lim _{k \rightarrow \infty} n_{k} / n_{k+1}=0$, then (2.26) and (2.27) hold with $K_{2}$ instead of $K_{1}$.

This theorem is proved in Section 4. It is also to be connected with Theorem 4.3. Theorem 2.1 together with $\left(2.2^{\prime}\right)$ show that in euclidian spaces, the partial sums of i.j.d. r.v.'s, when indexed on subsequences, can grow as slow as we want, it is enough to choose a subsequence of type defined in (2.2) with a function $\psi$ that grows very fast. The aim of our next statement is to show that the same property can happen in infinite dimensional spaces.

Theorem 2.10. Let $(T, \Theta)$ be a compact topological space. Assume there exists a sample continuous gaussian process $G=\{G(\omega, t), \omega \in \Omega, t \in T\}$ and set

$$
\forall s, t \in T, \quad \rho(s, t)=\left[E[G(s)-G(t)]^{2}\right]^{1 / 2} .
$$

Let $Y=\{Y(\omega, t), \omega \in \Omega, t \in T\}$ be a $\Theta$-separable random function satisfying 


$$
\begin{aligned}
& E \sup \left\{\left|\frac{Y(s)-Y(t)}{\rho(s, t)}\right|^{2},(s, t) \in T \otimes T\right\}<\infty, \\
& \forall t \in T, \quad E Y(t)=0 \quad \text { and } \quad E Y^{2}(t)<\infty .
\end{aligned}
$$

Let $(\xi, M, \varphi)$ be any triple defined in accordance with (2.1) and a sequence $Y_{1}, Y_{2}, \cdots$ of independent copies of $Y$. Then,

$$
P\left\{\left\{\frac{Y_{1}+\cdots+Y_{n}}{\sqrt{n} \varphi(n)}, n \in \mathfrak{\Xi}\right\} \text { is relatively compact in } \underline{C}(T)\right\}=1 .
$$

In other words, the random function $Y$ satisfies the compact law of the iterated logarithm in $\underline{C}(T)$ for any subsequence $\mathfrak{s}$.

This result is proved in Section 5 .

\section{§ 3. Characterization of the law of the iterated logarithm on subsequences}

Let us denote $W=\{W(t), 0 \leq t<\infty\}$ the usual brownian motion. In accordance with the notations $(2.1)$, let also $(\mathfrak{B}, M, \varphi)$ be a triple in which $\mathfrak{B}$ is a strictly increasing sequence of integers, $M>1$ and $\varphi$ defined by (2.1). Set,

$$
C(M)=\operatorname{median}(\sup \{W(t) / \sqrt{t}, 1 \leq t \leq M\}) .
$$

Lemma 3.1. For any triple $(\mathfrak{s}, M, \varphi)$,

$$
P\left\{\limsup _{\beta \ni n \rightarrow \infty} W(n) / \sqrt{n}-\varphi(n) \leq C(M)\right\}=1 .
$$

Furthermore,

$$
P\left\{\limsup _{8 \ni n \rightarrow \infty} W(n) / \sqrt{n} \varphi(n) \leq 1\right\}=1 .
$$

Proof. Fix $\varepsilon>0$ and set,

$$
\begin{aligned}
& \forall p \geq 1, \\
& \quad A_{p}=\left\{\sup \left(W(t) / \sqrt{t}, t \in \mathcal{B} \cap I_{k_{p}}\right)>\sqrt{2 \log (p+2)}+C(M)+\varepsilon\right\} .
\end{aligned}
$$

By applying Borell's inequality [1], and letting $\psi(x)=P\{N(0,1)>x\}$,

$$
\forall p \geq 1, \quad P\left\{A_{p}\right\} \leq \psi(\sqrt{2 \log (p+2)}+\varepsilon),
$$

so that, $P\left\{A_{p}, p\right.$ i.o. $\}=0$.

Q.E.D.

LEMMA 3.2. For any triple $(\xi, M, \varphi)$ and any sequence $Y_{1}, Y_{2}, \cdots$ of i.i.d. real r.v.'s satisfying $E Y_{1}=0$ and $E\left(Y_{1}\right)^{2}=1$, 


$$
P\left\{\limsup _{8 \ni n \rightarrow \infty} \frac{Y_{1}+\cdots+Y_{n}}{\sqrt{n} \varphi(n)} \leq 1\right\}=1 .
$$

Proof. By virtue of the Skohorod embedding scheme of partial sums (see e.g. [2], Theorem 13.6, p. 276), it is enough to prove

$$
P\left\{\limsup _{\S \ni n \rightarrow \infty} \frac{W\left(T_{1}+\cdots+T_{n}\right)}{\sqrt{n} \varphi(n)} \leq 1\right\}=1,
$$

where $T_{1}, T_{2}, \cdots$ is a sequence of nonnegative, independent, identically distributed r.v.'s with $E T_{1}=1$. Given $h>0$, we can choose $q$ large enough, so that, by virtue of the strong law of large numbers, the event

$$
A=\left\{\sup \left(\left|\frac{\sum_{i=1}^{n} T_{i}}{n}-1\right|, n \in \mathfrak{g}, n \geq q\right) \leq h\right\}
$$

has a probability greater than $1-h$. Fix $\varepsilon>0$ and set,

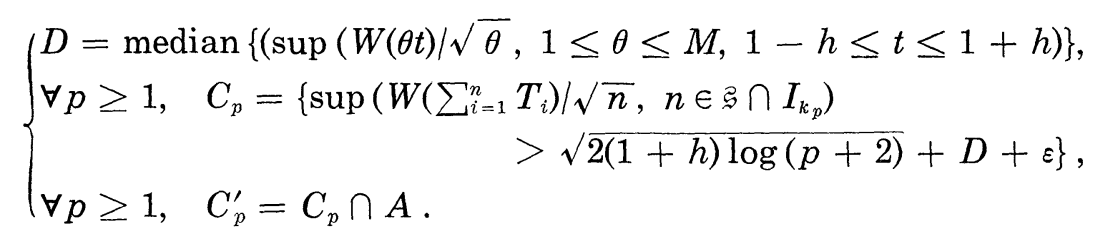

On $C_{p}^{\prime}$, one has $\sum_{i=1}^{n} T_{i}=\delta n$ for some $\delta \in[1-h, 1+h]$, and $n=\theta M^{k_{p}}$ for some $\theta \in[1, M]$. Thus,

$$
\begin{aligned}
C_{p}^{\prime} \subset C_{p}^{\prime \prime}=\left\{\operatorname { s u p } \left(\frac{W\left(\theta \delta M^{k_{p}}\right)}{\sqrt{\theta M^{k_{p}}}},\right.\right. & 1 \leq \theta \leq M, 1-h \leq \delta \leq 1+h) \\
& >\sqrt{2(1+h) \log (p+2)}+D+\varepsilon\},
\end{aligned}
$$

and

$$
\begin{aligned}
P\left\{C_{p}^{\prime \prime}\right\}=P\{\sup (W(\theta \delta) / \sqrt{\theta}, 1 & \leq \theta \leq M, 1-h \leq \delta \leq 1+h) \\
& >\sqrt{2(1+h) \log (p+2)}+D+\varepsilon\} .
\end{aligned}
$$

Since $\sup \left\{E(W(\theta \delta) / \sqrt{\theta})^{2}, 1 \leq \theta \leq M, 1-h \leq \delta \leq 1+h\right\}=1+h$, Borell's inequality again implies,

$$
P\left\{C_{p}^{\prime \prime}\right\} \leq \psi(\sqrt{2 \log (p+2)}+\varepsilon / \sqrt{1+h}) .
$$

Therefore,

$$
P\left\{C_{p}^{\prime \prime}, p \text { i.o. }\right\}=0
$$

and thus, 


$$
P\left\{\limsup _{8 \ni n \rightarrow \infty} \frac{W\left(\sum_{i=1}^{n} T_{i}\right)}{\sqrt{n} \varphi(n)} \leq \sqrt{1+h}\right\} \geq 1-h
$$

Since $h$ is arbitrary, we finally obtain,

$$
P\left\{\limsup _{8 \ni n \rightarrow \infty} \frac{W\left(\sum_{i=1}^{n} T_{i}\right)}{\sqrt{n} \varphi(n)} \leq 1\right\}=1 .
$$

Unlike the previous results, the proof of the next theorem involves a far more important work.

TheOREM 3.3. Let $(\mathfrak{s}, M, \varphi)$ be any triple defined in accordance with (2.1). Let also $X=\left\{X_{i}, i \geq 1\right\}$ be any sequence of i.i.d. real r.v.'s satisfying $E X_{1}=0$ and $E\left(X_{1}\right)^{2}=1$. Fix any $0<\rho<1$ and $0<\eta<1-\rho^{2}$ and let

$$
\begin{array}{r}
\left\{\begin{array}{l}
\mathfrak{s}(\rho, X)=\left\{n \in \mathfrak{S}: X_{1}+\cdots+X_{n} \geq \rho \sqrt{n} \varphi(n)\right\}, \\
\mathfrak{s}(\rho, \eta, X)=\left\{n \in \mathfrak{S}: X_{1}+\cdots+X_{n} \geq \rho \sqrt{n} \varphi(n)\right. \text { and }
\end{array}\right. \\
\varphi(n) \geq \Lambda(\mathfrak{s}) \sqrt{2 \eta \log \log n}\} .
\end{array}
$$

Then

$$
P\{\#(\xi(\rho, X))=\infty\}=1,
$$

and

$$
P\left\{\limsup _{8 \ni n \rightarrow \infty} \frac{X_{1}+\cdots+X_{n}}{\sqrt{n} \varphi(n)}=1\right\}=1
$$

Further

$$
P\left\{\Lambda(\mathfrak{s}(\rho, \eta, X)) \geq \Lambda(\mathfrak{g}) \sqrt{1-\rho^{2}}\right\}=1,
$$

and

$$
P\left\{\limsup _{\S \ni n \rightarrow \infty} \frac{X_{1}+\cdots+X_{n}}{\sqrt{2 n \log \log n}}=\Lambda(\mathfrak{s})\right\}=1
$$

Proof.

Step 1. By virtue of the Skohorod embedding scheme for partial sums, it is enough to prove the theorem when we replace $X_{1}+\cdots+X_{n}$ by $W\left(T_{1}+\cdots+T_{n}\right), n \geq 1$, where $T_{1}, T_{2}, \cdots$ is a sequence of i.i.d. nonnegative r.v.'s with $E T_{1}=1$. Let $h, \mu, \rho$ and $\rho_{0}$ be fixed in ]0, 1 [ with $\rho_{0}+\sqrt{h}<\rho$ 


$$
\varepsilon(h)=\operatorname{median}\{\sup (|W(\theta)-W(1)|, 1-h \leq \theta \leq 1+h)\},
$$

and for each integer $p \geq 1$,

$$
\left\{\begin{array}{l}
\varepsilon_{p}=\varepsilon(h)+\mu \sqrt{h}+\sqrt{2 h \log (p+2)} \\
A_{p}=\left\{\exists n \in \left[M^{p}, M^{p+1}\left[\cap \xi: X_{1}+\cdots+X_{n} \geq \rho_{0} \sqrt{n} \varphi(n)\right\}\right.\right. \\
A_{p}^{0}=\left\{X_{1}+\cdots+X_{n_{p}^{*}} \geq \rho_{0} \sqrt{2 n_{p}^{*} \log (p+2)}\right\} \\
A_{p}^{\prime}=\left\{\inf \left(W\left(\theta n_{p}^{*}\right) \sqrt{n_{p}^{*}}, 1-h \leq \theta \leq 1+h\right) \geq \rho_{0} \sqrt{2 \log (p+2)}\right\} \\
A_{p}^{\prime \prime}=\left\{W\left(n_{p}^{*}\right) / \sqrt{n_{p}^{*}}>\rho \sqrt{2 \log (p+2)}\right\} \\
A_{p}^{\prime \prime \prime}=\left\{\sup \left(\left|W\left(\theta n_{p}^{*}\right)-W\left(n_{p}^{*}\right)\right| \sqrt{n_{p}^{*}}, 1-h \leq \theta \leq 1+h\right) \leq \varepsilon_{p}\right\}
\end{array}\right.
$$

and choose $q$ large enough so that, by virtue of the strong law of large numbers, the event

$$
A=\left\{\sup \left(\left|\frac{\sum_{i=1}^{n} T_{i}}{n}-1\right|, n \geq q\right) \leq h\right\},
$$

has a probability greater than $1-h$.

Observe now, for any $m_{0}$ large enough and any $m \geq m_{0}$,

$$
\begin{aligned}
\sum_{p=m_{0}}^{m} I_{A_{k_{p}}} & \geq \sum_{p=m_{0}}^{m} I_{A_{p}^{0}}, \\
& \geq \sum_{p=m_{0}}^{m} I_{A_{p}^{\prime}} \cdot I_{A}, \\
& \geq \sum_{p=m_{0}}^{m} I_{A_{p}^{\prime \prime}} \cdot I_{A_{p}^{\prime \prime}} \cdot I_{A}, \\
& \geq\left[\sum_{p=m_{0}}^{m}\left(I_{A_{p}^{\prime \prime}}-I_{\left(A_{p}^{\prime \prime \prime}\right)}\right)\right] \cdot I_{A} .
\end{aligned}
$$

Suppose now,

$$
\sum_{p \geq 1} P\left\{\left(A_{p}^{\prime \prime \prime}\right)^{c}\right\}<\infty
$$

Then,

$$
P\left\{\exists m_{0}<\infty: \forall m \geq m_{0}, \sum_{p=m_{0}}^{m} I_{A_{p}^{0}} \geq \sum_{p=m_{0}}^{m} I_{A_{p}^{\prime \prime}} I_{A}\right\}=1 .
$$

By applying the classical Paley-Zygmund inequality for nonnegative square integrable r.v.'s: $P\{X \geq \lambda E X\} \geq[1-\lambda]^{2}[E X]^{2} / E(X)^{2}, 0 \leq \lambda \leq 1$, one has

$$
\begin{aligned}
P\left\{\sum_{p=m_{0}}^{m} I_{A_{p}^{\prime \prime}} \geq \lambda \sum_{p=m_{0}}^{m} P\left\{A_{p}^{\prime \prime}\right\}\right\} & {\left[\sum_{p=m_{0}}^{m} P\left\{A_{p}^{\prime \prime}\right\}\right]^{2} } \\
& \geq[1-\lambda]^{2} \frac{\sum_{\substack{p, q=m_{0} \\
p \neq q}} P\left\{A_{p}^{\prime \prime} \cap A_{q}^{\prime \prime}\right\}}{\sum_{p=m_{0}}^{m} P\left\{A_{p}^{\prime \prime}\right\}+\sum_{\substack{m \\
m}}^{m}} .
\end{aligned}
$$

Further, suppose,

(3.23) for any $0<h<1$, there exists $m_{0}=m_{0}(h)<\infty$, such that for every $m \geq m_{0}$ 


$$
\sum_{\substack{m_{0} \leq p, q \leq m \\ p \neq q}} P\left\{A_{p}^{\prime \prime} \cap A_{q}^{\prime \prime}\right\} \leq(1+h)\left[\sum_{p=m_{0}}^{m} P\left\{A_{p}^{\prime \prime}\right\}+\left(\sum_{p=m_{0}}^{m} P\left\{A_{p}^{\prime \prime}\right\}\right)^{2}\right]
$$

and

(3.24) for any $\left.\rho_{1} \in\right] \rho, 1\left[\right.$, there exists $m_{0}=m_{0}(\rho)<\infty$, and $m_{1}=m_{1}\left(m_{0}, \rho_{1}\right)$ $<\infty$, such that for every $m>\sup \left(m_{0}, m_{1}\right)$,

$$
\sum_{p=m_{0}}^{m} P\left\{A_{p}^{\prime \prime}\right\} \geq m^{1-\rho_{1}^{2}} .
$$

Then, putting together (3.19), (3.20), (3.21), (3.22), (3.23) and (3.24) leads to (3.25) for any strictly increasing sequence of integers $\Xi$ and any $0<\rho_{0}<$ $\rho_{1}<1$

$$
P\left\{\sum_{p=1}^{m} I_{A_{p}^{0}} \geq m^{1-\rho_{1}^{2}}, m \in \Xi, m \text { i.o. }\right\}=1 .
$$

This is our first step. We therefore must show (3.20), (3.23) and (3.24). We first prove (3.20). By using Borell's inequality,

$$
\begin{array}{rl}
\forall p \geq 1 & P\left\{\left(A_{p}^{\prime \prime \prime}\right)^{c}\right\} \\
\leq & P\left\{\sup \left(\left|W\left(\theta n_{p}^{*}\right)-W\left(n_{p}^{*}\right)\right| / \sqrt{n_{p}^{*}}, 1-h \leq \theta \leq 1\right)>\varepsilon_{p}\right\} \\
& +P\left\{\sup \left(\left|W\left(\theta n_{p}^{*}\right)-W\left(n_{p}^{*}\right)\right| \sqrt{n_{p}^{*}}, 1 \leq \theta \leq 1+h\right)>\varepsilon_{p}\right\}, \\
\leq & 2 \psi\left(\left[\varepsilon_{p}-\varepsilon(h)\right] / \sqrt{h}\right), \\
\leq & 2 \psi(\sqrt{2 \log (p+2)}+\mu) .
\end{array}
$$

Therefore, by applying Borel-Cantelli lemma, leads to (3.20). In order to obtain (3.23), we need the following classical estimate on gaussian distributions, (see e.g. [3], p. 269-270).

LEMMA 3.4. Let $U, V$ be jointly gaussian real r.v.'s satisfying $E U^{2}=$ $E V^{2}=1, E U=E V=0, E U V=r$ and let $\varepsilon>0$.

a) for any $x>0, y>0$ such that $r x y \leq \varepsilon$,

$$
P\{U>x, V>y\} \leq c(\varepsilon) P\{U>x\} P\{V>y\},
$$

where $\lim _{\varepsilon \rightarrow 0} c(\varepsilon)=1$;

b) for any $a \geq 0$, if $r \geq 0$,

$$
P\{\inf (U, V)>a\} \leq P\{U>a\} \psi\left(a \sqrt{\frac{1-r}{1+r}}\right) .
$$

Let $0<\alpha<\rho^{2}\left(1-M^{-1 / 2}\right) / 2$ be fixed and suppose first

a) $m_{0} \leq p<q \leq p+p^{\alpha}$,

then by Lemma 3.4 , 


$$
\begin{gathered}
\text { (3.27) } \quad P\left\{A_{p}^{\prime \prime} \cap A_{q}^{\prime \prime}\right\} \leq P\left\{A_{p}^{\prime \prime}\right\} \psi\left(\rho \sqrt{\left(1-M^{-1 / 2}\right) \log (p+2)}\right), \\
\text { since } \quad E\left[\frac{W\left(n_{p}^{*}\right) W\left(n_{q}^{*}\right)}{\sqrt{n_{p}^{*}} \sqrt{n_{q}^{*}}}\right] \leq M^{1-(q-p) / 2} \leq M^{-1 / 2}, \quad \text { if } q>p+1 .
\end{gathered}
$$

Thus,

$$
P\left\{A_{p}^{\prime \prime} \cap A_{q}^{\prime \prime}\right\} \leq P\left\{A_{p}^{\prime \prime}\right\}(p+2)^{-\rho^{2}(1-M-1 / 2) / 2},
$$

so that,

$$
\begin{aligned}
\sum_{p<q \leq p+p^{\alpha}} P\left\{A_{p}^{\prime \prime} \cap A_{q}^{\prime \prime}\right\} & \leq P\left\{A_{p}^{\prime \prime}\right\}\left\{[p+2]^{\alpha-\rho^{2}(1-M-1 / 2) / 2}+1\right\}, \\
& \leq(1+h) P\left\{A_{p}^{\prime \prime}\right\},
\end{aligned}
$$

once $m_{0}$ is sufficiently large.

b) $m_{0} \leq p \leq p+p^{\alpha}<q$.

Fix $\varepsilon \in] \alpha, 1\left[\right.$. Then assuming $m_{0}$ large enough, one has $q-p \geq q^{\varepsilon}$, so that

$$
\begin{aligned}
& \sup \left[\varphi\left(n_{p}^{*}\right) \varphi\left(n_{q}^{*}\right) E\left\{\frac{W\left(n_{p}^{*}\right) W\left(n_{q}^{*}\right)}{\sqrt{n_{p}^{*}} \sqrt{n_{q}^{*}}}\right\}, p>m_{0}, q>p+p^{\alpha}\right] \\
& \quad \leq 2 \sup \left[\left((\log p+2)(\log q+2) M^{2-(q-p)}\right)^{1 / 2}, p>m_{0}, q>p+p^{\alpha}\right] \\
& \quad \leq 2 \sup \left[(\log q+2) M^{\left(1-q^{\varepsilon}\right) / 2}, q \geq m_{0}\right] .
\end{aligned}
$$

By virtue of Lemma 3.4, this one leads to

$$
P\left\{A_{p}^{\prime \prime} \cap A_{q}^{\prime \prime}\right\} \leq(1+h) P\left\{A_{p}^{\prime \prime}\right\} P\left\{A_{q}^{\prime \prime}\right\},
$$

once $m_{0}$ is sufficiently large, that we suppose; thus (3.23) is now established. As for (3.24), fix $1>\rho^{\prime \prime}>\rho^{\prime}>\rho$. For $m_{0}$ large enough,

$$
\begin{aligned}
\sum_{p=m_{0}}^{m} P\left\{A_{p}^{\prime \prime}\right\} & \geq \frac{1}{\sqrt{2 \pi}} \sum_{p=m_{0}}^{m} \frac{1}{1+\rho \sqrt{2 \log (p+2)}}[p+2]^{-\rho^{2}}, \\
& \geq \sum_{p=m_{0}}^{m}[p+2]^{-\left(\rho^{\prime}\right) 2}, \\
& \geq\left\{[m+3]^{1-\left(\rho^{\prime}\right)^{2}}-\left[m_{0}+2\right]^{1-\left(\rho^{\prime}\right) 2}\right\} /\left[1-\left(\rho^{\prime}\right)^{2}\right], \\
& \geq m^{1-\left(\rho^{\prime \prime}\right) 2},
\end{aligned}
$$

once $m>m_{1}=m_{1}\left(m_{0}, \rho^{\prime}, \rho^{\prime \prime}\right)$. Hence (3.24) holds and consequently our first step, which is (3.25) is now established. Letting $\Xi=N$ and $\rho_{0}$ tending to 1 in (3.25) leads to $\left(3.13^{\prime}\right)$.

Step 2. In this step we prove (3.14). Obviously, there is no loss when assuming $\Lambda(\mathfrak{g})>0$. Fix $0<\delta^{\prime}<\Lambda(\mathfrak{g})^{2}$, and set

$$
\Xi=\left\{m \in N: k_{m} \leq m^{1 / \hat{o}^{\prime}}\right\}
$$


Since

$$
\begin{gathered}
\left.\Lambda(\mathfrak{g})^{2}=\limsup _{m \rightarrow \infty}[\log m)\right] /\left[\log k_{m}\right]>\delta^{\prime}, \\
\sharp(\Xi)=\infty .
\end{gathered}
$$

Fix also $0<\eta<1-\rho_{1}^{2}$. Then, according to (3.25), with probability one, there are infinitely many integers $m \in \Xi$, such that,

(3.34) $A_{k_{p}}$ occurs for at least $m^{1-\rho_{1}^{2}}-m^{\eta}$ integers $p$ in the interval $\left[m^{\eta}, m\right]$, since, one has

$$
\sum_{1 \leq p \leq m} I_{A_{k_{p}}} \leq m^{\eta}+\sum_{m \eta<p \leq m} I_{A_{k_{p}}} .
$$

For such integers, by definition of $A_{k_{p}}$, one has

$$
S_{n}(X) \geq \rho_{0} \sqrt{n} \varphi(n) \quad \text { for some } n \in \mathfrak{g} \cap I_{k_{p}},
$$

and

$$
\varphi(n)=\sqrt{2 \log (p+2)} .
$$

But $p \geq m^{\eta}, n \leq M^{k_{p}+1} \leq M^{k_{m}+1}$ and $k_{m} \leq m^{1 / \hat{o}^{\prime}} ;$ hence

$$
\begin{aligned}
\log (p+2) & \geq \log \left[2+\left(\frac{\log (n / M)}{\log M}\right)^{\delta^{\prime} \eta}\right], \\
& \geq[\log \log n] \delta^{\prime} \eta^{\prime},
\end{aligned}
$$

for any $0<\eta^{\prime}<\eta$, once $n$ is sufficiently large, namely, once $m$ is large enough. Thus, we have

$$
\varphi(n) \geq \sqrt{2 \delta^{\prime} \eta^{\prime} \log \log n} .
$$

On the whole, with probability one, there are infinitely many integers $m$ such that

$$
\left\{\begin{array}{l}
\text { there exists at least } m^{1-\left(\rho_{1}\right)^{2}}-m^{\eta} \text { integers } p \in\left[m^{\eta}, m\right] \text { such that } \\
S_{n}(X) \geq \rho_{0} \sqrt{n} \varphi(n) \text { and } \varphi(n) \geq \sqrt{2 \delta^{\prime} \eta^{\prime} \log \log n} \text { for some } n \in \mathfrak{S} \cap I_{k_{p}} .
\end{array}\right.
$$

Letting $\delta^{\prime} \eta^{\prime}=\Lambda^{2}(\mathfrak{s}) \eta^{\prime \prime}$, gives

$$
P\left\{\sum_{1 \leq p \leq m} I_{\left\{\vec{\beta}\left(\rho_{0}, \eta^{\prime \prime}, X\right) \cap I_{k_{p}}\right\}} \geq m^{1-\left(\rho_{1}\right)^{2}}-m^{\eta}, m \in \Xi, m \text { i.o. }\right\}=1 .
$$

Since

$$
\sum_{1 \leq p \leq m} I_{\left\{\beta\left(\rho_{0}, \eta^{\prime \prime}, X\right) \cap I_{k_{p}}\right\}}=\sum_{1 \leq i \leq k_{m}} I_{\left\{\overline { q } ( \rho _ { 0 } , \eta ^ { \prime \prime } , X ) \cap \left[M^{i}, M^{i+1}[\}\right.\right.},
$$

(3.37) together with the definition of $\Lambda(\mathfrak{g})$ imply

$$
P\left\{\Lambda\left(\mathfrak{S}\left(\rho_{0}, \eta^{\prime \prime}, X\right)\right) \geq \sqrt{1-\left(\rho_{1}\right)^{2}} \Lambda(\mathfrak{g})\right\}=1 .
$$

This one easily establishes (3.14). 
Step 3. We now prove (3.15). Let $X^{i}=\left\{X_{n}^{i}, n \geq 1\right\}, i=1,2$, be two independent copies of the sequence $X=\left\{X_{n}, n \geq 1\right\}$, and define

$$
c_{1}=\max \left\{\rho \sqrt{1-\rho^{2}}, 0<\rho<1\right\}=1 / 2 .
$$

From (3.14) and (3.39),

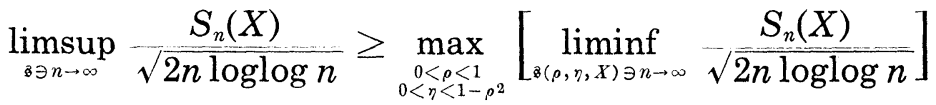

$$
\begin{aligned}
& \geq(1 / 2) \Lambda(\mathfrak{s})
\end{aligned}
$$

almost surely. This is the first step. We now apply (3.40) and (3.14) simultaneously. We first observe, for every $0<\alpha<1$,

$$
\begin{gathered}
\limsup _{\beta \ni n \rightarrow \infty} \frac{S_{n}\left(\alpha X^{1}+X^{2} \sqrt{1-\alpha^{2}}\right)}{\sqrt{2 n \log \log n}} \geq \limsup _{\delta\left(\rho, \tau, X^{2}\right) \ni n \rightarrow \infty} \frac{S_{n}\left(\alpha X^{1}+X^{2} \sqrt{\left.1-\alpha^{2}\right)}\right.}{\sqrt{2 n \log \log n}}, \\
\geq\left[\liminf _{\left\{\left(\rho, \eta, X^{2}\right) \ni n \rightarrow \infty\right.} \frac{S_{n}\left(X^{2}\right)}{\sqrt{2 n \log \log n}}\right] \sqrt{1-\alpha^{2}} \\
+\alpha\left[\limsup _{\delta\left(\rho, \eta, X^{2}\right) \ni n \rightarrow \infty} \frac{S_{n}\left(X^{1}\right)}{\sqrt{2 n \log \log n}}\right],
\end{gathered}
$$

almost surely, and thus

$$
\left.\geq\left[\sqrt{1-\alpha^{2}(\rho} \sqrt{\eta}\right)+\frac{\alpha}{2} \sqrt{1-\rho^{2}}\right] \Lambda(\text { ) }),
$$

by virtue of (3.40) (and 3.14) since $X^{1}$ and $X^{2}$ are independent. We put

$$
\begin{aligned}
& \forall n \geq 1, \\
& \quad c_{n}=\max \left\{\left[\rho \sqrt{1-\alpha^{2}}+\alpha c_{n-1}\right] \sqrt{1-\rho^{2}}, 0<\rho<1,0<\alpha<1\right\} .
\end{aligned}
$$

From (3.42), assuming that $X$ is gaussian,

$$
P\left\{\limsup _{\Re \ni n \rightarrow \infty} \frac{S_{n}(X)}{\sqrt{2 n \log \log n}} \geq c_{2} \Lambda(\mathfrak{g})\right\}=1,
$$

and, by repeating the same argument,

$$
P\left\{\limsup _{\mathfrak{g} \ni n \rightarrow \infty} \frac{S_{n}(X)}{\sqrt{2 n \log \log n}} \geq \sup _{p}\left(c_{p}\right) \Lambda(\mathfrak{g})\right\}=1 .
$$

Now, the lower bound in (3.25) will be deduced from the study of the sequence $\left\{c_{n}, n \geq 1\right\}$. It is easy to check that the maximum of

$$
\varphi_{\rho, n}(\alpha)=\left\{\rho \sqrt{1-\alpha^{2}}+\alpha c_{n-1}\right\} \sqrt{1-\rho^{2}}
$$

is reached at the value 


$$
\alpha(\rho, n)=c_{n-1} / \sqrt{\rho^{2}+\left(c_{n-1}\right)^{2}}
$$

and

$$
f_{n}(\rho)=\varphi_{\rho, n}(\alpha(\rho, n))=\sqrt{\left[1-\rho^{2}\right]\left[\left(c_{n-1}\right)^{2}+\rho^{2}\right]} .
$$

Further, $f_{n}(\cdot)$ has unique maximum on $[0,1]$ at the value $\rho(n)=$ $\sqrt{\left[1-\left(c_{n-1}\right)^{2}\right] / 2}$. Its corresponding value defines $c_{n}$ and finally, one has

$$
\forall n \geq 1, \quad c_{n}=\left[1+\left(c_{n-1}\right)^{2}\right] / 2 .
$$

It is now easy to see that $c_{n}$ increases to 1 when $n$ tends to infinity. This gives the lower bound in (3.15) when $X$ is gaussian.

We now must prove this in general. First, it is quite clear that $\Lambda(\mathfrak{g})=\Lambda\left(\mathfrak{g}^{*}\right)$. Replacing $\mathfrak{g}$ by $\mathfrak{g}^{*}$, our result becomes

$$
P\left\{\limsup _{p \rightarrow \infty} \frac{W\left(n_{p}^{*}\right)}{\sqrt{2 n_{p}^{*} \log \log n_{p}^{*}}} \geq \Lambda(\mathfrak{s})\right\}=1 .
$$

By virtue of the Skohorod embedding scheme and (3.18), for any integer $p$ and $0<h<1$,

$$
\begin{aligned}
P\{A & \left.\cap\left\{\left|W\left(\sum_{i=1}^{n_{p}^{*}} T_{i}\right)-W\left(n_{p}^{*}\right)\right| / \sqrt{n_{p}^{*}}>\sqrt{2 h \log \log n_{p}^{*}}+\varepsilon(h)+\mu \sqrt{h}\right\}\right\} \\
\leq P\{\sup (|W(\theta)-W(1)|, 1-h \leq \theta \leq 1+h) & \left.\quad>\sqrt{2 h \log \log n_{p}^{*}}+\varepsilon(h)+\mu \sqrt{h}\right\}, \\
\leq & \quad \psi\left(\sqrt{2 \log \log n_{p}^{*}}+\mu\right), \quad \text { (by applying Borell's inequality) } \\
& \leq 2 \psi\left(\sqrt{2 \log [p(\log M)]}+\mu, \quad \text { (since } n_{p}^{*} \geq M^{k_{p}} \geq M^{p}\right) .
\end{aligned}
$$

Therefore, by applying Borel-Cantelli lemma and letting $h$ tend to 0 ,

$$
P\left\{\lim _{p \rightarrow \infty} \frac{\left|W\left(\sum_{i=1}^{n_{p}^{*}} T_{i}\right)-W\left(n_{p}^{*}\right)\right|}{\sqrt{2 n_{p}^{*} \log \log n_{p}^{*}}}=0\right\}=1 .
$$

Putting together (3.47) and (3.49) leads to

$$
P\left\{\limsup _{p \rightarrow \infty} \frac{S_{n_{p}^{*}}(X)}{\sqrt{2 n_{p}^{*} \log \log n_{p}^{*}}} \geq \Lambda(\mathfrak{g})\right\}=1,
$$

which produces the lower bound in (3.15).

We now turn to the upper bound of (3.15). Set,

$$
L(X)=\limsup _{8 \in n \rightarrow \infty} \frac{S_{n}(X)}{\sqrt{2 n \log \log n}} .
$$

By 0-1 law, $L(X)$ is a number and there is no loss when assuming $L(X)$ 
$>0$. Let $0<L^{\prime}<L(X)$ be fixed. Then for some random subsequence $\left\{n_{j}, j \geq 1\right\}$ of $\mathfrak{s}$

$$
\forall j \geq 1, \quad L^{\prime} \leq \frac{S_{n_{j}}(X)}{\sqrt{2 n_{j} \log \log n_{j}}} .
$$

Define the random sequence of integers $\left\{p_{j}, j \geq 1\right\}$ such that

$$
\forall j \geq 1, \quad n_{j} \in I_{k_{p_{j}}} .
$$

Fix $h>0$ arbitrary. By Lemma 3.2, for all large enough $j$,

$$
S_{n_{j}}(X) \leq \sqrt{(1+h) n_{j} \varphi\left(n_{j}\right)}=\sqrt{(1+h) n_{j} 2 \log \left(p_{j}+2\right)},
$$

so that,

$$
\begin{aligned}
L^{\prime} & \leq\left[\frac{\log \left(p_{j}+2\right)}{\log \log n_{j}}(1+h)\right]^{1 / 2}, \\
& \leq\left[\frac{(1+h) \log \left(p_{j}+2\right)}{\log \left[(\log M) k_{p_{j}}\right]}\right]^{1 / 2}, \\
& \leq\left[\frac{(1+h) \log \left\{2+\sum_{i=1}^{k_{p_{j}}} \delta_{i}(\mathcal{S}, M)\right\}}{\log \left[(\log M) k_{p_{j}}\right]}\right]^{1 / 2} .
\end{aligned}
$$

Therefore,

$$
L^{\prime} \leq \sqrt{1+h} \limsup _{p \rightarrow \infty}\left[\frac{\log \sum_{i=1}^{p} \delta_{i}(\mathfrak{g}, M)}{\log p}\right]^{1 / 2}
$$

Letting $L^{\prime}$ tend to $L$ and $h$ tend to 0 gives the conclusion. Thus (3.15) is proved, and the proof of the Theorem 3.3 is now complete. Q.E.D.

The first part of the above proof is very classical in the study of upper or lower classes of gaussian sequences; part 2 and part 3 are the original parts of the proof.

Lemma 3.5. For any triple $(\mathfrak{s}, M, \varphi)$ defined in accordance with (2.1) and any sequence $X=\left\{X_{\imath}, i \geq 1\right\}$ of i.i.d. real r.v.'s satisfying $E X_{1}=0$ and $E\left(X_{1}\right)^{2}<\infty$,

$$
P\left\{C\left(\left\{S_{n}(X) / \sqrt{n} \varphi(n), n \in \mathfrak{\xi}\right\}\right)=\left[-\sqrt{E\left(X_{1}\right)^{2}}, \sqrt{E\left(X_{1}\right)^{2}}\right]\right\}=1 .
$$

Proof. The proof is very simple. First, by homogeneity there is no loss when assuming $E\left(X_{1}\right)^{2}=1$. Then, let $\delta \geq 1$ be fixed. We associate to it the following subsequence of $\mathfrak{g}^{*}$,

$$
\mathfrak{\xi}_{\delta}^{*}=\left\{n_{\left[p^{\delta}\right]}^{*}, p \geq 1\right\} .
$$


The corresponding sequence $k_{p}^{\delta}=k_{p}\left(\xi_{\tilde{\delta}}^{*}\right), p \geq 1$, trivially satisfies,

$$
\forall p \geq 1, \quad k_{p}^{o}=k_{\left[p^{\tilde{o}}\right]},
$$

so that the corresponding function $\varphi_{\delta}$ also satisfies

$$
\forall n \in \mathfrak{F}_{\dot{\delta}}^{*}, \quad \varphi_{\delta}(n)=\sqrt{2 \log (p+2)} \text { iff } n \in \mathfrak{F}_{\dot{\delta}}^{*} \cap I_{k\left[p^{\delta}\right]},
$$

and therefore $\varphi_{\delta}(n) \approx \varphi(n) \sqrt{\delta}$ as $n$ tends to infinity along the sequence 踪・ By applying $\left(3.13^{\prime}\right)$, one obtains

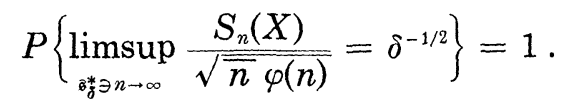

This one easily implies (3.57).

Q.E.D.

The next lemma shows the necessity of the condition $E\left(X_{1}\right)^{2}<\infty$. The proof uses a classical argument on truncated r.v.'s.

Lemma 3.6. Let $(\mathfrak{B}, M, \varphi)$ be any triple defined in accordance with (2.1). Let also $X=\left\{X_{i}, i \geq 1\right\}$ be a sequence of i.i.d. real r.v.'s. Then,

$$
P\left\{\limsup _{\bar{s} \ni n \rightarrow \infty} \frac{S_{n}(X)}{\sqrt{n} \varphi(n)}=1\right\}=1,
$$

if, and only if,

$$
E X_{1}=0 \text { and } E\left(X_{1}\right)^{2}=1 .
$$

Proof. There is just the "only if" part of the assertion to prove. First, assume that $X_{1}$ is a symmetric r.v., and let $c>0$ fixed. Then,

$$
X_{1}^{\prime}=X_{1} I_{\left\{\left|X_{1}\right| \leq c\right\}}-X_{1} I_{\left\{\left|X_{1}\right|>c\right\}},
$$

has same distribution as $X_{1}$. Let $X^{\prime}=\left\{X_{i}^{\prime}, i \geq 1\right\}$ be a sequence of independent copies of $X_{1}^{\prime}$. By writing (3.60) for $X$ and $X^{\prime}$, then using triangular inequality, one obtains,

$$
P\left\{\limsup _{\nexists \ni n \rightarrow \infty} \frac{S_{n}\left(X I_{\{|X| \leq c\}}\right)}{\sqrt{n} \varphi(n)} \leq 1\right\}=1
$$

By $\left(3.13^{\prime}\right)$,

$$
P\left\{\limsup _{8 \ni n \rightarrow \infty} \frac{S_{n}\left(X I_{\{|X| \leq c)}\right)}{\sqrt{n} \varphi(n)}=\sqrt{E\left\{X_{1}^{2} I_{\left\{\left|X_{1}\right| \leq c\right\}}\right\}}\right\}=1
$$

so that,

$$
E\left\{X_{1}^{2} I_{\left\{\left|X_{1}\right| \leq c\right\}}\right\} \leq 1
$$


and finally $E X_{1}^{2} \leq 1$. But, by using again (3.13') and (3.60), this, necessarily implies $E X_{1}^{2}=1$. If $X_{1}$ is not symmetric, let $X^{\prime}$ be an independent copy of $X$, and set $Y=X-X^{\prime}$. Writing (3.60) for $X$ and $X^{\prime}$, and using triangular inequality, leads to

$$
P\left\{\limsup _{8 \ni n \rightarrow \infty} \frac{S_{n}(Y)}{\sqrt{n} \varphi(n)} \leq 2\right\}=1 .
$$

Therefore, $E\left[X_{1}-X_{1}^{\prime}\right]^{2} \leq 4$, and by Corollary 2 (M. Loève, Probability theory, $3^{d}$ ed., p. 246),

$$
E\left|X_{1}-\mu X_{1}\right|^{2} \leq 8,
$$

where $\mu X_{1}$ denotes a median of $X_{1}$. This implies $E\left|X_{1}\right|^{2}<\infty$. By applying (3.13') and (3.60), one obtains

$$
E\left(X_{1}\right)^{2}=1
$$

Further, $E X_{1}=m<\infty$. The centering of $X_{1}$ follows from the strong law of large numbers, since

a.s. $|m|=\lim _{\beta \ni n \rightarrow \infty}\left|\frac{S_{n}(X)}{n}\right| \leq \limsup _{\beta \ni n \rightarrow \infty} \frac{\left|S_{n}(X)\right|}{\sqrt{n} \varphi(n)} \cdot \limsup _{\beta \ni n \rightarrow \infty} \frac{\varphi(n)}{\sqrt{n}}=0 . \quad$ Q.E.D.

Proof of Theorem 2.1.

a) if $m=1$. This is given by $\left(3.13^{\prime}\right)$ in Theorem 3.3, Lemmas 3.5 and 3.6 .

b) if $1 \leq m<\infty$. The sufficiency results from the proof of Lemma 2 in [5], in which the sequence of normalization constants $\{\sqrt{2 n \log \log n}$, $n \geq 3\}$ does not matter except the fact that it is needed to control the case $m=1$.

As for the necessity, let $f_{i}, f_{i, i}, i, j=1, \cdots m$, be linear forms on $\boldsymbol{R}^{m}$ defined by $f_{i}(x)=x^{i}$, and $f_{i, j}(x)=x^{i}+x^{j}$ for every $x=\left(x^{1}, \cdots, x^{m}\right) \in \boldsymbol{R}^{m}$. By applying Lemma 3.5 to the sequences $\left\{f_{i, j}\left(S_{n}(X)\right), n \in \mathfrak{F}\right\}, i, j=1, \cdots m$, one obtains

$$
\begin{array}{ll}
\forall i, j=1, \cdots m, \quad & E f_{i, j}\left(X_{1}\right)^{2}=E\left[X_{1}^{i}+X_{1}^{j}\right]^{2}=2, \\
& E f_{i}\left(X_{1}\right)^{2}=E\left[X_{1}^{i}\right]^{2}=1, \\
& E f_{i}\left(X_{1}\right)=E X_{1}^{\imath}=0,
\end{array}
$$

which easily allows to conclude.

Q.E.D.

Proof of Theorem 2.3. As far as this is just the assertion (3.15) of Theorem 3.3, it is already proved.

Q.E.D. 
Proof of Theorem 2.8 .

a) Lower bound. Fix $0<\rho_{0}<\rho_{1} \leq 1$, and set,

$$
\begin{cases}\forall p \geq 1, \quad k_{p}^{1}=k_{p}^{1}\left(\mathfrak{I}_{1}, M\right), \quad\left(k_{p}=k_{p}(\mathfrak{F}, M)\right), \\ \left.\forall n \in \mathfrak{J}_{1}, \quad \varphi_{1}(n)=\varphi\left(\mathfrak{I}_{1}, M, n\right), \quad \text { (using notations }(2.1)\right), \\ \forall p \geq 1, \quad A_{p}=\left\{\exists n \in \mathfrak { Z } _ { 1 } \cap \left[M^{p}, M^{p+1}\left[: S_{n}(X) \geq \rho_{0} \sqrt{n} \varphi_{1}(n)\right\} .\right.\right.\end{cases}
$$

Since $\xi_{1}$ is a subsequence of $\xi$, then $\left\{k_{p}^{1}, p \geq 1\right\}$ is a subsequence of $\left\{k_{p}\right.$, $p \geq 1\}$, and

$$
\forall p \geq 1, \quad k_{p}^{1}=k_{c(p)},
$$

where $c: N \rightarrow N$ is strictly increasing $(c(p) \geq p$, for every $p)$.

There is no loss when assuming $\Lambda\left(\mathfrak{g}, M, \mathfrak{I}_{1}\right)>0$. Observe that

$$
\begin{aligned}
\Lambda\left(\mathfrak{s}, M, \mathfrak{g}_{1}\right) & =\limsup _{m \rightarrow \infty}\left[\frac{\log m}{\log \#\left\{i \leq k_{m}^{1}: \mathfrak{B} \cap\left[M^{i}, M^{i+1}[\neq \varnothing\}\right.\right.}\right]^{1 / 2}, \\
& =\limsup _{m \rightarrow \infty}\left[\frac{\log m}{\log c(m)}\right]^{1 / 2} .
\end{aligned}
$$

Fix $0<\delta<\Lambda^{2}\left(\mathfrak{s}, M, \mathfrak{g}_{1}\right)$, and set

$$
\Xi=\left\{m \in N: c(m) \leq m^{1 / \delta}\right\} .
$$

Then $\sharp(\Xi)=\infty$. Further, by (3.25)

$$
P\left\{\sum_{i=1}^{m} I_{A k_{p}^{1}} \geq m^{1-\left(\rho_{1}\right)^{2}}, m \in \Xi, m \text { i.o. }\right\}=1 .
$$

Fix $0<\eta<1-\left(\rho_{1}\right)^{2}$. With probability one, there are infinitely many $m \in \Xi$ such that

$$
A_{k_{p}^{1}} \text { occurs for at least } m^{1-\left(\rho_{1}\right)^{2}}-m^{\eta} \text { integers } p \in\left[m^{\eta}, m\right] .
$$

For these integers one has

and

$$
S_{n}(X) \geq \rho_{0} \sqrt{n} \varphi_{1}(n) \quad \text { for some } n \in \mathfrak{I}_{1} \cap I_{k_{p}^{1}}(M),
$$

But $m^{\eta} \leq p \leq m$, and $M^{k_{p}^{1}}=M^{k_{c(p)}} \leq n<M^{k_{p}^{1}+1}=M^{k_{c(p)+1}}$, so that

$$
\varphi(n)=\sqrt{2 \log (c(p)+2)} \leq \sqrt{2 \log (c(m)+2)} .
$$

Thus,

$$
\begin{aligned}
\varphi_{1}(n) & \geq \sqrt{2 \log \left(2+m^{\eta}\right)}, \\
& \geq \sqrt{2 \log \left(2+c(m)^{\delta \eta}\right)}, \\
& \geq \sqrt{\delta \eta^{\prime}} \varphi(n),
\end{aligned}
$$


for any $0<\eta^{\prime}<\eta$, once $n$ is large enough. Therefore, with probability one, there are infinitely many $m \in \Xi$ such that

there exist at least $m^{1-\left(\rho_{1}\right)^{2}}-m^{\eta}$ integers $p \in\left[m^{\eta}, m\right]$ for which $S_{n}(X) \geq \rho_{0} \sqrt{n} \varphi_{1}(n)$ and $\varphi_{1}(n) \geq \sqrt{\delta \eta^{\prime}} \varphi(n)$ for some $n \in \mathfrak{B}_{1} \cap I_{k_{p}^{1}}$.

By arguing along the lines (3.36)-(3.38), one obtains for,

$$
\begin{aligned}
& \quad \mathfrak{J}_{1}\left(\rho_{0}, \eta^{\prime \prime}, X\right) \\
& \quad=\left\{n \in \mathfrak{I}_{1}: S_{n}(X) \geq \rho_{0} \sqrt{n} \varphi_{1}(n) \text { and } \varphi_{1}(n) \geq \sqrt{\eta^{\prime \prime}} \Lambda\left(\mathfrak{g}, M, \mathfrak{g}_{1}\right) \varphi(n)\right\}, \\
& P\left\{\Lambda\left(\mathfrak{g}, M, \mathfrak{I}_{1}\left(\rho_{0}, \eta^{\prime \prime}, X\right)\right) \geq \sqrt{1-\left(\rho_{1}\right)^{2}} \Lambda\left(\mathfrak{g}, M, \mathfrak{I}_{1}\right)\right\}=1,
\end{aligned}
$$

where we put $\delta \eta^{\prime}=\eta^{\prime \prime} \Lambda^{2}\left(\mathfrak{s}, M, \mathfrak{S}_{1}\right)$. Next, we conclude by following the same scheme of proof as in step 3 of the proof of Theorem 3.3.

b) Upper bound. Again there is no loss when assuming that

$$
L(X)=\limsup _{\mathbb{8}_{1} \ni n \rightarrow \infty} \frac{S_{n}(X)}{\sqrt{n} \varphi(n)}>0 .
$$

Fix $0<L^{\prime}<L(X)$, and let $h>0$. Then for some random subsequence $\left\{n_{j}, j \geq 1\right\}$ of $\mathfrak{i}_{1}$,

$$
\forall j \geq 1, \quad L^{\prime} \leq S_{n_{j}}(X) / \sqrt{n_{j}} \varphi\left(n_{j}\right) .
$$

One defines two random sequences of integers $\left\{q_{j}, j \geq 1\right\}$ and $\left\{p_{j}, j \geq 1\right\}$ such that

By Lemma 3.2,

$$
\forall j \geq 1, \quad n_{j} \in I_{k_{p_{j}}^{1}}(M) \cap I_{k_{q_{j}}}(M)
$$

$$
S_{n_{j}}(X) \leq(1+h) \sqrt{2 n_{j} \log \left(p_{j}+2\right)}
$$

for all $j$ large enough, and $\varphi\left(n_{j}\right)=\sqrt{2 \log \left(q_{j}+2\right)}$. Thus,

$$
L^{\prime} \leq(1+h)\left[\frac{\log \left(p_{j}+2\right)}{\log \left(q_{j}+1\right)}\right]^{1 / 2},
$$

for all $j$ large enough. This one easily leads to the result by letting $L^{\prime}$ tend to $L(X)$ and $h$ tend to 0 .

Q.E.D.

\section{§4. Strassen's laws of the iterated on subsequences}

First, we recall the following lemma due to J. Kuelbs ([9], p. 247-248).

Lemma 4.1. 1) Let $B$ a separable Banach space. Let $\left\{Y_{k}, k \geq 1\right\}$ be a sequence of $B$-valued random variables and assume $\mu$ is a mean zero 
Gaussian measure on $B$. Let $K$ denote the unit ball of the reproducing Hilbert space $H_{\mu}$ of $\mu$. If,

$$
L\left(\left(Y_{k}\right), \mu\right)=b_{k} \quad(k \geq 1),
$$

where $\sum_{k=1}^{\infty} b_{k}<\infty$, and $L$ is the Prohorov metric for probability measures on $(B,\|\cdot\|)$. Then,

$$
P\left\{\omega: \lim _{n \rightarrow \infty} d\left(\frac{Y_{n}(\omega)}{\sqrt{2 \log n}}, K\right)=0\right\}=1,
$$

where $d(x, A)=\inf \{\|x-y\|, y \in A\}$.

2) If $\left(Y_{k}\right)=\mu$ for every $k$, and

$$
\left.\lim _{\substack{m \rightarrow \infty \\ k-m \rightarrow \infty}} E\left\{E\left[f\left(Y_{k}\right) \mid \underline{F}_{m}\right]\right\}^{2}\right)=0,
$$

for every $f \in B^{*}$ where $\underline{F}_{m}=\underline{F}\left\{Y_{k}, k \leq m\right\}$; then

$$
P\left\{\omega: C\left(\left\{\frac{Y_{n}(\omega)}{\sqrt{2 \log n}}, n \geq 1\right\}\right)=K\right\}=1 .
$$

Let $W$ be a 1-dimensional brownian motion and define for every integer $n$

$$
\zeta_{n}(t)=\frac{W(n t)}{\sqrt{n}} \quad(0 \leq t \leq 1) .
$$

Clearly each $\left\{\zeta_{n}(t), 0 \leq t \leq 1\right\}$ is a brownian motion on $[0,1]$ and it induces a Wiener measure $\mu$ on $\underline{C}([0,1])$. Further, it is well known that the reproducing kernel Hilbert space of $\mu$ is

$$
H_{\mu}=\left\{f \in \underline{C}([0,1]): f(t)=\int_{0}^{t} g(u) d u \text { where } \int_{0}^{1} g^{2}(s) d s<\infty\right\},
$$

with inner product $\left\langle f_{1}, f_{2}\right\rangle=\int_{0}^{1} f_{1}^{\prime}(u) f_{2}^{\prime}(u) d u$ and hence

$$
K=\left\{f \in \underline{C}([0,1]): f(t)=\int_{0}^{t} g(u) d u \text { where } \int_{0}^{1} g^{2}(u) d u \leq 1\right\} .
$$

Let now $(\mathfrak{s}, M, \varphi)$ be defined in accordance with (2.1) and set

$$
\forall p \geq 1, \quad \zeta_{p}^{*}=\zeta_{n_{p}^{*}}
$$

LemMA 4.2. For any triple $(\mathfrak{s}, M, \varphi)$

a) $P\left\{\lim _{n \leftarrow \infty} d\left(\frac{\zeta_{n_{p}^{*}}}{\sqrt{2 \log (p+2)}}, K\right)=0\right\}=1$, 
b) $\left.P\left\{C\left\{\frac{\zeta_{n_{p}^{*}}}{\sqrt{2 \log (p+2)}}, p \geq 1\right\}\right)=K\right\}=1$.

Proof. We mimic Kuelbs's proof in [9] p. 249-251. Since $L\left(\left(\zeta_{p}^{*}\right), \mu\right)=0$, a) is easily deduced from the first part of Lemma 4.1. To prove b) if $f \in \underline{C}^{*}([0,1])$ with $f(x)=\int_{0}^{1} x(t) d F(t)$, for $x \in \underline{C}([0,1])$, then

$$
\begin{aligned}
E\left\{f\left(\zeta_{p+q}^{*}\right) \mid \underline{F}_{p}\right\} & =E\left\{\int_{0}^{1} \frac{W\left(n_{p+q}^{*} t\right)}{\sqrt{n_{p+q}^{*}}} d F(t) \mid \underline{F}_{p}\right\}, \\
= & E\left\{\int_{0}^{n_{p}^{*} / n_{p+q}^{*}} \frac{W\left(n_{p+q}^{*} t\right)}{\sqrt{n_{p+q}^{*}}} d F(t)+\int_{n_{p}^{*} / n_{p+q}^{*}}^{1} \frac{W\left(n_{p+q}^{*} t\right)}{\sqrt{n_{p+q}^{*}}} d F(t) \mid \underline{F}_{p}\right\} \\
= & \int_{0}^{n_{p}^{*} / n_{p+q}^{*}} \frac{W\left(n_{p+q}^{*} t\right)}{\sqrt{n_{p+q}^{*}}} d F(t)+\int_{n_{p}^{*} / n_{p+q}^{*}}^{1} \frac{W\left(n_{p}^{*}\right)}{\sqrt{n_{p+q}^{*}}} d F(t) .
\end{aligned}
$$

Hence,

$$
\begin{aligned}
E\left\{E\left[f\left(\zeta_{p}^{*}\right) \mid \underline{E}_{p}\right]^{2}\right)= & \frac{1}{n_{p+q}^{*}} \int_{0}^{n_{p}^{*} / n_{p+q}^{*}} \int_{0}^{n_{p}^{*} / n_{p+q}^{*}} \min \left[n_{p+q}^{*} s, n_{p+q}^{*} t\right] d F(s) d F(t) \\
& +\frac{2}{n_{p+q}^{*}} \int_{0}^{n_{p}^{*} / n_{p+q}^{*}} \int_{n_{p}^{*} / n_{p+q}^{*}}^{1} \min \left[n_{p}^{*}, n_{p+q}^{*} t\right] d F(s) d F(t) \\
& +\frac{n_{p}^{*}}{n_{p+q}^{*}}\left(\int_{n_{p}^{*} / n_{p+q}^{*}+}^{1} d F(t)\right)^{2},
\end{aligned}
$$

and (4.3) holds since $\lim _{q \rightarrow \infty} \sup _{p \geq 1} n_{p}^{*} / n_{p+q}^{*}=0$. Thus, the second part of Lemma 4.1 gives b).

Q.E.D.

(4.6') Lemma 4.2 then holds for any subsequence $\mathfrak{S}^{\prime} \subset \mathcal{B}$ such that $\#\left(\mathfrak{s}^{\prime} \cap I_{k_{p}}\right)=1$, for every $p$. This simple observation will be afterwards convenient.

ThEOREM 4.3. For any triple $(\mathfrak{\xi}, M, \varphi)$ defined in accordance with (2.1),

a) $P\left\{\lim _{8 \ni n \rightarrow \infty} d\left(\frac{\zeta_{n}}{\varphi(n)}, K\right)=0\right\}=1$,

b) $P\left\{C\left(\left\{\frac{\zeta_{n}}{\varphi(n)}, n \in \mathfrak{g}\right\}\right)=K\right\}=1$.

Proof. Fix $h>0$ and define

$$
\begin{cases}\forall p \geq 1, & J\left(\mathfrak{g}, k_{p}, k\right)=\mathfrak{S} \cap\left[M^{k_{p}}(1+(k-1) h), M^{k_{p}}(1+k h)[,\right. \\ & \text { where } k \in \Delta(\mathfrak{S}, p) \text { and } \\ \forall p \geq 1, & \Delta(\mathfrak{g}, p)=\left\{1 \leq j \leq(M-1) / h: J\left(\mathfrak{S}, k_{p}, j\right) \neq \varnothing\right\},\end{cases}
$$




$$
\left\{\begin{array}{l}
\beta=4 E\{\sup \{|W(r)|, 1 \leq r \leq M\}\}, \\
\forall p \geq 1, \quad \forall k \in \Delta(\mathfrak{I}, p), \quad n_{n, k}^{*} \text { is the first point of } J\left(\mathfrak{s}, k_{p}, k\right), \\
\forall p \geq 1, \quad \forall k \in \Delta(\mathfrak{g}, p), \quad \zeta_{p, k}^{*}=\zeta_{n_{p}^{*}, k}^{*} .
\end{array}\right.
$$

Fix $k \in[1,(M-1) / h]$ and set for each $p$ such that $k \in \Delta(\mathfrak{s}, p)$,

$$
A_{p}^{k}=\left\{\exists n \ni J\left(\mathfrak{g}, k_{p}, k\right):\left\|\zeta_{n}-\zeta_{p, k}^{*}\right\| \geq \sqrt{2 h \log (p+2)}+2 \beta\right\} .
$$

Observe now, for $n \in J\left(\xi, k_{p}, k\right)$ and $0 \leq \theta \leq 1$,

$$
\begin{aligned}
E\left|\frac{W(n \theta)}{\sqrt{n}}-\frac{W\left(n_{p, k}^{*} \theta\right)}{\sqrt{n_{p, k}^{*}}}\right|^{2} & =2 \theta\left(1-n_{p, k}^{*} / n\right), \\
& \leq 2 h,
\end{aligned}
$$

and,

$$
\begin{aligned}
& E\left\{\sup \left\{\left\|\zeta_{n}-\zeta_{p, k}^{*}\right\|, n \in J\left(\Xi, k_{p}, k\right)\right\}\right\} \\
& \quad \leq E\left\{\sup \left\{\left\|\zeta_{n}\right\|, n=M^{k_{p}} \lambda, 1 \leq \lambda \leq M\right\}\right\}+E\left\{\left\|\zeta_{p, k}^{*}\right\|\right\} \\
& \quad \leq \beta / 2 .
\end{aligned}
$$

By applying Borell's inequality,

$$
\forall p \geq 1, \quad P\left\{A_{p}^{k}\right\} \leq 2 \psi(\sqrt{2 \log (p+2)}+\beta / \sqrt{h}),
$$

so that, for each $k \in[1,(M-1) / h]$,

$$
P\left\{\left\{\exists n \in J\left(\xi, k_{p}, k\right):\left\|\zeta_{n}-\zeta_{p, k}^{*}\right\|>\sqrt{2 h \log (p+2)}+2 \beta\right\}, p \text { i.o. }\right\}=0 .
$$

Therefore,

$$
P\left\{\limsup _{p \rightarrow \infty} \sup \left\{\frac{\left\|\zeta_{n}-\zeta_{p, k}^{*}\right\|}{\varphi(n)}, n \in J\left(\mathfrak{g}, k_{p}, k\right)\right\} \leq \sqrt{h}\right\}=1 .
$$

But Lemma 4.2 and remark $\left(4.6^{\prime}\right)$ imply,

$$
\forall k \in[1,(M-1) / h], \quad P\left\{\lim _{p \rightarrow \infty} d\left(\zeta_{p, k}^{*} / \sqrt{2 \log (p+2)}, K\right)=0\right\}=1,
$$
and,

(4.15) $\forall k \in[1,(M-1) / h], \quad P\left\{C\left(\left\{\zeta_{p, k}^{*} ! \sqrt{2 \log (p+2)}, p \geq 1\right\}\right)=K\right\}=1$.

Combining (4.13) with (4.14), then letting $h$ tend to 0

$$
P\left\{\lim _{\S \ni n \rightarrow \infty} d\left(\zeta_{n} / \varphi(n), K\right)=0\right\}=1 .
$$

Combining (4.15) with (4.16), 


$$
P\left\{C\left(\left\{\zeta_{n} / \varphi(n), n \in \mathfrak{g}\right\}\right)=K\right\}=1 .
$$

Lemma 4.4. For any triple $(\xi, M, \varphi)$ defined in accordance with (2.1) such that

$$
\lim _{k \rightarrow \infty} n_{k} / n_{k+1}=1, \quad\left(\mathfrak{S}=\left\{n_{k}, k \geq 1\right\}\right),
$$

and for any sequence $X=\left\{X_{i}, i \geq 1\right\}$ of i.i.d. real r.v.'s satisfying $E X_{1}=0$ and $E\left(X_{1}\right)^{2}=1$, one has

$$
P\left\{\lim _{j \rightarrow \infty}\left\|\frac{\eta_{j}}{\sqrt{n_{j}}}-\zeta_{n_{j}}\right\| / \varphi\left(n_{j}\right)=0\right\}=1,
$$

where $\eta_{j}, j \geq 1$, is defined in (2.24) relatively to $X$ and $\mathfrak{g}$.

Proof. By virtue of the Skohorod embedding scheme for partial sums, it is enough to prove the Lemma 4.4 when replacing $X_{i}(i \geq 1)$, by

$$
\tilde{X}_{i}=W\left(T_{1}+\cdots+T_{i}\right)-W\left(T_{1}+\cdots+T_{i-1}\right) \quad(i \geq 1),
$$

where $W$ is a 1-dimensional brownian motion and $T_{1}, T_{2}, \ldots$ a sequence of nonnegative i.i.d. r.v.'s satisfying $E T_{1}=1$. Define, with the convention $n_{0}=0$,

$$
\begin{aligned}
& \forall \theta \geq 0, \text { if } n_{k} \leq \theta \leq n_{k+1} \text { for some } k \geq 0, \\
& \tilde{\eta}(\theta)=\tilde{S}_{n_{k}}\left[1+\left(n_{k}-\theta\right) /\left(n_{k+1}-n_{k}\right)\right]+\tilde{S}_{n_{k+1}}\left[\left(\theta-n_{k}\right) /\left(n_{k+1}-n_{k}\right)\right], \\
& \text { where } \tilde{S}_{n_{k}}=\sum_{i=1}^{n_{k}} \tilde{X}_{i} \quad(k \geq 1) .
\end{aligned}
$$

Then,

$$
|\tilde{\eta}(t)-W(t)| \leq \max \left\{\left|\tilde{S}_{n_{k}}-W(t)\right|,\left|\tilde{S}_{n_{k+1}}-W(t)\right|\right\},
$$

if $n_{k} \leq t \leq n_{k+1}$. Fix $h>0$. By the strong law of large numbers as well as assumption (4.19), we can choose an integer $q$ large enough in order that

$$
A=\left\{\sup \left\{\left|\left(\sum_{i=1}^{n_{k}} T_{i}\right) / n_{k}-1\right|, k \geq q\right\} \leq h\right\}
$$

satisfies

$$
P\{A\} \geq 1-h
$$

and further,

$$
\sup \left\{\left[n_{k+1}-n_{k}\right] / n_{k}, k \geq q\right\} \leq h .
$$

For $\omega \in A, k \geq q$ and $n_{k} \leq t \leq n_{k+1}$, 


$$
\left|\sum_{i=1}^{n_{k}} T_{i}-t\right| \leq 2 h n_{k}
$$

Set

$$
\forall \theta \in[0,1], \quad \forall j \geq 1, \quad \tilde{\eta}_{j}(\theta)=\tilde{\eta}\left(\theta n_{j}\right) / \sqrt{n_{j}} .
$$

If $\theta \geq n_{q} / n_{j}, j \geq q$,

$$
\begin{aligned}
\left|\tilde{\eta}_{j}(\theta)-\frac{W\left(\theta n_{j}\right)}{\sqrt{n_{j}}}\right| & \leq \max \left\{\left|\frac{W(s)-W\left(\theta n_{j}\right)}{\sqrt{n_{j}}}\right|,\left|\theta n_{j}-s\right| \leq 2 h n_{j}, 0 \leq \theta \leq 1\right\}, \\
& \leq \max \left\{\left|\frac{W\left(u n_{j}\right)-W\left(\theta n_{j}\right)}{\sqrt{n_{j}}}\right|, 0 \leq \theta \leq 1,|u-\theta| \leq 2 h\right\} .
\end{aligned}
$$

Let $p$ be fixed and $n_{j} \in I_{k_{p}} \cap \mathfrak{\xi}$; one has on $A$,

$$
\begin{aligned}
& \sup \left\{\left|\tilde{\eta}_{j}(\theta)-\zeta_{n_{j}}(\theta)\right|, n_{q} / n_{j} \leq \theta \leq 1\right\} \\
& \quad \leq \sup \left\{\left|W\left(M^{k_{p}} \alpha \gamma\right)-W\left(M^{k_{p}} \theta \gamma\right)\right| \mid M^{k_{p} / 2},\right. \\
& \quad 1 \leq \gamma \leq M, 0 \leq \theta \leq 1,|\theta-\gamma| \leq 2 h\},
\end{aligned}
$$

and on $\Omega$,

(4.28) $\sup \left\{\left|\tilde{\eta}_{j}(\theta)-\zeta_{n_{j}}(\theta)\right|, 0 \leq \theta \leq n_{q} / n_{j}\right\}$

$$
\leq M^{-k_{p} / 2}\left[\max \left\{\left|\tilde{S}_{j}\right|, j \leq q+1\right\}+\max \left\{|W(s)|, 0 \leq s \leq n_{q}\right\}\right],
$$

so that

$$
P\left\{\lim _{j \rightarrow \infty} \sup \left\{\left|\tilde{\eta}_{j}(\theta)-\zeta_{n_{j}}(\theta)\right|, 0 \leq \theta \leq n_{q} / n_{j}\right\}=0\right\}=1
$$

Set

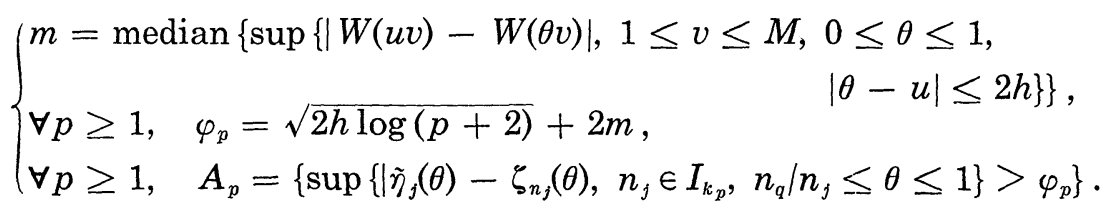

By using Borell's inequality again,

$$
P\left\{A_{p} \cap A\right\} \leq 2 \psi(\sqrt{2 \log (p+2)}+m / \sqrt{2 h}),
$$

so that, $P\left\{A_{p} \cap A, p\right.$ i.o. $\}=0$, and combining this with (4.23),

$$
P\left\{\limsup _{j \rightarrow \infty} \sup \left\{\left|\tilde{\eta}_{j}(\theta)-\zeta_{n_{j}}(\theta)\right| / \varphi\left(n_{j}\right), n_{q} / n_{j} \leq \theta \leq 1\right\} \leq \sqrt{2 h}\right\}=1 .
$$

From (4.30) and (4.33),

$$
P\left\{\limsup _{j \rightarrow \infty}\left\|\tilde{\eta}_{j}-\zeta_{n_{j}}\right\| / \varphi\left(n_{j}\right) \leq \sqrt{2 h}\right\} \geq 1-h .
$$


This achieves the proof by letting $h$ tend to 0 .

Q.E.D.

As an easy consequence, one has, by putting together Lemma 4.4 and Theorem 4.3

Corollary 4.5. For any triple $(\mathfrak{\xi}, M, \varphi)$ be defined in accordance with (2.1), such that (4.19) holds, and for any sequence $X=\left\{X_{i}, i \geq 1\right\}$ of i.i.d. real r.v.'s satisfying $E X_{1}=0$ and $E\left(X_{1}\right)^{2}=1$, one has

$$
P\left\{\lim _{j \rightarrow \infty} d\left(\eta_{j} / \varphi\left(n_{j}\right), K\right)=0\right\}=1,
$$

and

$$
P\left\{C\left(\left\{\eta_{j} / \varphi\left(n_{j}\right), j \geq 1\right\}\right)=K\right\}=1 .
$$

Proof of Theorem 2.9.

a) if $\lim _{k \rightarrow \infty} n_{k} / n_{k+1}=1$. This is already proved by Corollary 4.5.

b) if $\lim _{k \rightarrow \infty} n_{k} / n_{k+1}=0$. This is easily deduced from

$$
P\left\{\lim _{N \rightarrow \infty} \sup \left(\frac{\left|S_{n_{k}}\right|}{\sqrt{ } n_{N} \varphi\left(n_{N}\right)}, 1 \leq k \leq N-1\right)=0\right\}=1
$$

and the fact that the sequence of subdivisions $\left\{n_{k} / n_{N}, 1 \leq k \leq N\right\}$ tends to $\{0,1\}$.

Q.E.D.

\section{$\S 5$. The law of the iterated logarithm on subsequences for random functions}

As explained in Section 2, the main goal of this section is to prove that the behavior of partial sums of i.i.d. r.v.'s taking value in some infinite dimensional space $B$, can be as small as we want, like in euclidian spaces, when indexed on subsequences. This is the aim of the Theorem 2.10 , that we are going to prove. We will use the following classical exponential bound for martingales.

Lemma 5.1. Let $M_{n}=\sum_{i=1}^{n} d_{i},(n \geq 1)$, be a real-valued martingale satisfying

$$
\forall i \geq 1, \quad\left|d_{i}\right| \leq \alpha_{i} \quad \text { a.s. }
$$

Then,

$$
\forall t \geq 0, \quad P\left\{\left|M_{n}\right| \geq t\right\} \leq 2 \exp \left\{-t^{2} / 2\left[\sum_{i=1}^{n} \alpha_{i}^{2}\right]\right\}
$$


Proof of Theorem 2.10. We first assume that $Y$ is a symmetric r.v. For the clarity we denote $\left(\Omega_{Y}, \mathscr{A}_{Y}, P_{Y}\right)$ the basic probability space of the sequence $Y_{1}, Y_{2}, \cdots$. Let $g_{1}, g_{2}, \cdots$ be independent $\mathscr{N}(0,1)$ r.v.'s defined on another probability space $\left(\Omega_{g}, \mathscr{A}_{g}, P_{g}\right)$. Let also $\varepsilon_{1}, \varepsilon_{2}, \cdots$ be independent Rademacher r.v.'s defined on a third probability space $\left(\Omega_{\varepsilon}, \mathscr{A}_{\varepsilon}, P_{\varepsilon}\right)$. The corresponding symbols of integration are denoted $E_{Y}, E_{g}$ and $E_{\varepsilon}$ respectively. We now use an argument due to V.V. Yurinskii [19], which will be the first tool of the proof. Let $\underline{F}_{i}=\sigma\left\{\varepsilon_{1}, \cdots, \varepsilon_{i}\right\}$ for every $i \geq 1$. Then

$$
\left\|\sum_{k=1}^{n} Y_{k} \varepsilon_{k}\right\|-E_{\varepsilon}\left\|\sum_{k=1}^{n} Y_{k} \varepsilon_{k}\right\|=\sum_{k=1}^{n} d_{i}
$$

where

$$
\forall i \geq 1, \quad d_{i}=E^{F_{i}}\left\{\left\|\sum_{k=1}^{n} Y_{k} \varepsilon_{k}\right\|\right\}-E^{F_{i-1}}\left\{\left\|\sum_{\substack{k=1 \\ k \neq i}}^{n} Y_{k} \varepsilon_{k}\right\|\right\},
$$

and thus, $\left|d_{i}\right| \leq 2\left\|Y_{i}\right\|$, for every $i \geq 1$.

By applying Lemma 5.1 conditionally,

$$
\begin{gathered}
\forall t \geq 0, \quad P_{\varepsilon}\left\{\left|\left\|\sum_{i=1}^{n} Y_{i} \varepsilon_{i}\right\|-E_{\varepsilon}\left\|\sum_{i=1}^{n} Y_{i} \varepsilon_{i}\right\|\right|>t\right\}, \\
\leq 2 \exp \left\{-\frac{t^{2}}{8 \sum_{i=1}^{n}\left\|Y_{i}\right\|^{2}}\right\} .
\end{gathered}
$$

We now need to control $E_{\varepsilon}\left\{\left\|\sum_{i=1}^{n} Y_{i} \varepsilon_{i}\right\|\right\}$. By using the easy fact that $\left\{g_{i}, i \geq 1\right\}$ and $\left\{\left|g_{i}\right| \varepsilon_{i}, i \geq 1\right\}$ are identically distributed, one has by Jensen inequality,

$$
E_{\varepsilon}\left\{\left\|\sum_{i=1}^{n} Y_{i} \varepsilon_{i}\right\|\right\} \leq \sqrt{\frac{\pi}{2}} E_{g}\left\{\left\|\sum_{i=1}^{n} Y_{i} g_{i}\right\|\right\}
$$

Further

$$
\begin{aligned}
\forall(s, t) \in T \otimes T, \quad\left[E_{g} \mid\right. & \left.\left.\sum_{k=1}^{n} g_{k}\left(Y_{k}(s)-Y_{k}(t)\right)^{1 / 2}\right|^{2}\right], \\
& \leq\left[\sum_{k=1}^{n}\left(Y_{k}(s)-Y_{k}(t)^{2}\right]^{1 / 2},\right. \\
& \leq \rho(s, t)\left[\sum_{i=1}^{n} D_{i}^{2}\right]^{1 / 2},
\end{aligned}
$$

where $D_{1}, D_{2}, \cdots$ is a sequence of independent copies of $\sup \{[(Y(s)-$ $Y(t) / \rho(s, t),(s, t) \in T \otimes T\}$. Since we have assumed that $G$ is sample continuous, the classical integrability properties of gaussian processes (see e.g. [4]), together with Slepian's lemma (see again [4], Theorem 2.1.2) and results in [13], imply

$$
\forall n \geq 1, \quad E_{\varepsilon}\left\{\left\|\sum_{i=1}^{n} Y_{i} \varepsilon_{i}\right\|\right\} \leq B\left[\sum_{i=1}^{n} D_{i}^{2}\right]^{1 / 2},
$$


where the constant $B<\infty$ only depends on $T$ and $\rho$ and tends to 0 with $\operatorname{diam}(T, \rho)$, Let $c>\max \left(1, E D_{1}^{2}\right)$ be fixed and

$$
\left.A=\sup \left\{\sum_{i=1}^{n} D_{i}^{2} / n, n \geq q\right\} \leq c\right\},
$$

where we choose $q$ large enough so that, by virtue of the strong law of large numbers.

$$
P\{A\} \geq 1 / 2
$$

On $A$, one has, (using (5.7)),

$$
\sup \left\{\left[E_{\varepsilon}\left\{\left\|\sum_{i=1}^{n} Y_{i} \varepsilon_{i}\right\|\right\}\right] / \sqrt{ } n, n \geq q\right\} \leq B c .
$$

Let now $(\xi, M, \varphi)$ be any triple defined according to (2.1). Choose $s>0$ such that,

$$
\forall p \geq 1, \quad s \sqrt{\left[2 n_{p}^{*} \log (p+2)\right] / M}>B c \sqrt{n_{p}^{*}}+3 \sqrt{n_{p}^{*} c \log (p+2)} .
$$

Then, applying Levy's inequality conditionally to $P_{\varepsilon}$,

$$
\begin{array}{r}
P_{Y} \otimes P_{\varepsilon}\left\{\left\{\sup \left[\left\|\sum_{i=1}^{n} Y_{i} \varepsilon_{i}\right\| / \sqrt{2 n \log (p+2)}, n \in \mathfrak{B} \cap I_{k_{p}}\right]>s\right\} \cap A\right\} \\
\leq 2 E_{Y}\left\{I_{A} \cdot P_{\varepsilon}\left\{\left\|\sum_{i=1}^{n_{p}^{*}} Y_{i} \varepsilon_{i}\right\|>s \sqrt{\left[2 n_{p}^{*} \log (p+2)\right]} / M\right\}\right\},
\end{array}
$$

and by (5.4),

$$
\begin{aligned}
& \leq 4 E_{Y}\left\{I_{A} \cdot \exp \left(-\frac{9 c n_{p}^{*} \log (p+2)}{8 \sum_{1 \leq i \leq n_{p}^{*}} D_{i}^{2}}\right)\right\}, \\
& \leq 4 P_{Y}\{A\} \exp \left\{-\frac{9}{8} \log (p+2)\right\},
\end{aligned}
$$

so that, by using Borel-Cantelli lemma and 0-1 law,

$$
P_{Y}\left\{\sup \left\{\frac{\left\|S_{n}(Y)\right\|}{\sqrt{n} \varphi(n)}, n \in \mathfrak{\xi}\right\}<\infty\right\}=1,
$$

then, by (5.7) and usual conclusion drawn from the inequality of J. Hoffman-Jørgensen [8], p. 164-165.

$$
E_{Y}\left\{\sup \left\{\frac{\left\|S_{n}(Y)\right\|}{\sqrt{n} \varphi(n)}, n \in \xi\right\}\right\}<\infty .
$$

We now can drop the assumption of symmetry, by using classical inequality of symmetrization. The conclusion is obtained by applying, as usual, the closed graph theorem and arguing as along the lines following (4.13) in [10].

Q.E.D. 


\section{§6. Conclusion}

In this work, several problems are solved, and in the same time, some others are raised. One can summarize them as follows:

Problem 1 (Conjecture): is any unbounded sequence of integers $\mathfrak{Z}$ a natural basis of the integers (i.e. $\mathfrak{g}^{(r)}=N$ for some finite $r$ ), if, and only if, $\Lambda\left(2^{\mathfrak{z}}\right)>0$ ?, (assuming $1 \in \mathfrak{Z}$ ).

Problem 2: identify the set of cluster points $C\left(\left\{\eta_{N} /\left[\sqrt{n_{N}} \varphi\left(n_{N}\right)\right], N \geq 1\right\}\right)$ in full generality. A partial answer is brought by the Theorem 2.9.

Problem 3: extend Theorem 2.1 in any Banach space. The recent characterization of this property when $\mathcal{S}=N$ [10], reducing it to check the same property in probability, is certainly a good basis. Nevertheless the classical condition

$$
E\left\{\|X\|^{2} / \log \log \|X\|\right\}<\infty,
$$

which is needed to satisfy this property, is no longer necessary when indexing partial sums on subsequences. This brings a serious complication in order to truncate the r.v.'s, that is a necessary step in the proof given in [10], since the corresponding condition cannot be expressed in term of moment of $X$.

Acknowledgement. This work was partially done during my stay, from October 1986 to March 1987, as visiting member, at the Courant Institute of Mathematical Sciences of New-York University, and I am indepted to Professor S.R.S. Varadhan for several useful and stimulating comments.

\section{REFERENCES}

[1] Borell, C., The Brunn-Minkowski inequality in Gauss space, Invent. Math., 30 (1975), 207-216.

[2] Breiman, L., Probability. Addison Wesley, 1968.

[ 3 ] Chung, K. L., Erdos, P. and Sirao, T., On the Lipschitz's conditions for Brownian motion, J. Math. Soc. Japan, 11 (1959), 263-274.

[4] Fernique, X., Régularité des trajectoires des fonctions aléatoires gaussiennes, Ecole d'été de Probabilités de Saint-Flour 1974, Lecture Notes in Math., 480 (1975), 1-96. Springer, Berlin.

[5] Finkelstein, H., The law of the iterated logarithm for empirical distributions, Ann. of Math. Stat., 42 (1971), 607-615.

[6] Gut, A., The law of the iterated on subsequences, Publ. Uppsala University, 1983. 
[ 7 ] Hartman, P. and Wintner, A., On the law of the iterated logarithm, Amer. J. Math., 63 (1941), 169-176.

[ 8 ] Hoffman-Jørgensen, J., Sums of independent Banach space valued random variables, Studia Math., 52 (1974), 159-186.

[9] Kuelbs, J., The law of the iterated logarithm and related strong convergence theorems for Banach space valued random variables, Ecole d'été de Probabilité de Saint-Flour 1975. Lecture Notes in Math., 539 (1976), 224-314. Springer, Berlin.

[10] Ledoux, M. and Talagrand, M., Characterization of the law of the iterated logarithm in Banach spaces, Ann. of Prob., 16, (1988), 1242-1264.

[11] Strassen, V., An invariance principle for the law of the iterated logarithm, Z. Wahrsch. verw. Geb., 3 (1964), 211-226.

[12] Strassen, V., A converse to the law of the iterated logarithm, Z. Wahrsch. verw. Geb., 4 (1966), 265-268.

[13] Talagrand, M., Regularity of gaussian processes, Acta Math., 159 (1987), 99-149.

[14] Torrang, I., The law of the iterated logarithm - cluster points of deterministic and random subsequences, Prob. Math. Stat., 8 (1987), 133-141.

[15] Vaughan, R. C., The Hardy-Littlewood method, Cambridge University Press, 1981.

[16] Weber, M., La loi du logarithme itéré sur les sous-suites, Comptes Rendus Acad. Sci. Paris, 303 Sér. 1 (1986), 77-80.

[17] Weber, M., La loi du logarithme itéré sur toute sous-suite, caractérisations, C.R. Acad. Paris, 305 (1987), 835-840.

[18] Weber, M., The law of the iterated logarithm for subsequences in Banach spaces, Prob. in Banach spaces VII, (1988), to appear in Birkhäuser ed., Progress in Prob. and Stat.

[19] Yurinskii, V. V., Exponential bound for large deviations, Theor. Prob., Appl., 19 (1974), 154-155.

Université Louis Pasteur.

Uer de Mathématiques et Informatique

7, rue René Descartes

67084 Strasbourg Cedex

France 